\title{
Color unified dynamical axion
}

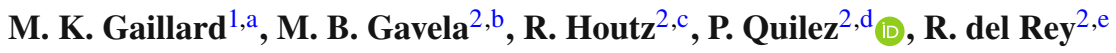 \\ ${ }^{1}$ Department of Physics and Theoretical Physics Group, Lawrence Berkeley Laboratory, University of California, Berkeley, California 94720, \\ USA \\ ${ }^{2}$ Departamento de Física Teórica and Instituto de Física Teórica, IFT-UAM/CSIC, Universidad Autónoma de Madrid, Cantoblanco, 28049 Madrid, \\ Spain
}

Received: 31 May 2018 / Accepted: 30 October 2018 / Published online: 24 November 2018

(C) The Author(s) 2018

\begin{abstract}
We consider an enlarged color sector which solves the strong $\mathrm{CP}$ problem via new massless fermions. The spontaneous breaking of a unified color group into QCD and another confining group provides a source of naturally large axion mass $m_{a}$ due to small size instantons. This extra source of axion mass respects automatically the alignment of the vacuum, ensuring a low-energy $\mathrm{CP}$-conserving vacuum. The mechanism does not appeal to a $Z_{2}$ "mirror" copy of the $\mathrm{SM}$, nor does it require any fine-tuning of the axion-related couplings at the unification scale. There is no very light axion and uncharacteristically the lighter spectrum contains instead sterile fermions. The axion scale $f_{a}$ can be naturally brought down to a few $\mathrm{TeV}$, with an exotic spectrum of colored pseudoscalars lighter than this scale, observable at colliders exclusively via strong interactions. The $\left\{m_{a}, f_{a}\right\}$ parameter space which allows a solution of the strong $\mathrm{CP}$ problem is thus enlarged well beyond that of invisible axion models.
\end{abstract}

\section{Contents}

1 Introduction ................ 2

$2 \mathrm{SU}(6)$ color unification . . . . . . . . . . . 3

3 The realistic color unified theory: $S U(6) \times S U\left(3^{\prime}\right)$. 4

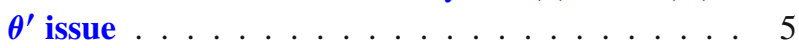

3.1 Model I: adding a massless fermion charged under $S U\left(3^{\prime}\right) \ldots \ldots \ldots \ldots \ldots$

3.1.1 Running of the coupling constants . . . 6

3.1.2 Confinement of $S U(3)_{\text {diag }}$ and pseudoscalar anomalous couplings to the confining interactions . . . . . . . . .

6
3.1.3 The impact of small-size instantons on the dynamical axion mass . . . . . . . 8

3.1.4 Small-size instantons with small Yukawa couplings 9

How light can the axion that couples to SSI become? . . . . . . . . . . 10

Solution to the strong CP problem . . . . . 10

Computation of the pseudoscalar mass matrix: $\eta_{\chi}^{\prime}, \eta_{\psi}^{\prime}, \eta_{\mathrm{QCD}}^{\prime}$ and light spectrum . . . . 11

Low energy spectrum and observable effects . . 12

3.1.5 Small-size instantons with $\mathcal{O}(1)$ Yukawa couplings . . . . . . . . . 12

Solution to the strong CP problem . . . . . 13

Low energy spectrum and observable effects . . 13

3.2 Model II: Addition of a second $\Delta$ scalar. . . . . 13

3.2.1 Running of the coupling constants . . . 14

3.2.2 Confinement of $S U(3)_{\text {diag }}$ and pseudoscalar anomalous couplings to the confining interactions. . . . . . . . . 14

3.2.3 Impact of small-size instantons on the dynamical axion mass . . . . . . . 15

Small-size instantons of the spontaneously broken CUT . . . . . . . . . . . 15

Solution to the strong CP problem . . . . . 15 Computation of the pseudoscalar mass matrix: $a, \eta_{\psi}^{\prime}, \eta_{\mathrm{QCD}}^{\prime}$ and light spectrum $\ldots . .16$

4 Phenomenological and cosmological limits on the lightest exotic states . . . . . . . . . . . . 16

4.1 Collider observable signals . . . . . . . . . 16

4.1.1 Color-octet pions from $S U(3)_{\text {diag }}$ confinement . 17

4.1.2 Dynamical axion and exotic fermions . . 17

4.1.3 The axion coupled to SSI . . . . . . . 17

4.2 Cosmological and gravitational aspects . . . . . 17

4.2.1 Stable particles and cosmological structures 17

4.2.2 The question of gravitational quantum effects 18

5 Conclusions . . . . . . . . . . . . 18

References . . . . . . . . . . . . . . . . . . 19 


\section{Introduction}

Phenomenological analyses based on chiral perturbation theory and supported by lattice computations indicate that all Standard Model (SM) quarks have non-zero masses. This disfavors the solution to the strong CP problem via one massless SM quark, which automatically guarantees a $U(1)$ axial invariance at the classical level. The interesting possibility of having a massless up quark in the microscopic theory which appears as massive at QCD scales due to non-perturbative instanton contributions [1] does not seem to be realized in nature, even if this option is not completely excluded [2-7].

It is still possible to solve the strong $\mathrm{CP}$ problem using massless fermions if the SM up quark is massive. The idea is to enlarge the SM gauge group with a new confining sector [8-10], whose scale is much larger than that of the QCD group, $S U(3)_{c}$. Extra massless quarks charged under both QCD and the new confining sector may realistically solve the problem [11]. A new spectrum of confined states results.

The term "axion" denotes any (pseudo)Goldstone boson (pGB) of a global chiral $U(1)$ symmetry which is exact at the classical level but has anomalous couplings to the field strength of a confining group. ${ }^{1}$ Axions are characteristic of solutions to the strong CP problem based on an anomalous $U$ (1) axial symmetry, usually called Peccei-Quinn (PQ) symmetry [12]. When the number of axions in a given theory outnumbers the total number of distinct instanton-induced scales other than QCD to which they couple, one (or more) light axions remain. Axions which are not elementary but composed of fermions are typical of theories with an enlarged confining sector and are often referred to as "dynamical" or composite axions. Very heavy dynamical axions made out of massless fermions will typically acquire the bulk of their mass from the largest instanton-induced scale $\Lambda$ to which they exhibit anomalous couplings,

$m_{a}^{2} f_{a}^{2} \sim \Lambda^{4}$.

For a very light axion coupled only to QCD, the mixing with the $\eta^{\prime}$ pseudoscalar is relevant instead, and that axion obeys the usual relation $[13,14]$

$m_{a}^{2} f_{a}^{2} \sim m_{\pi}^{2} f_{\pi}^{2} \frac{m_{u} m_{d}}{\left(m_{u}+m_{d}\right)^{2}}$,

where $m_{\pi}, f_{\pi}, m_{u}, m_{d}$ denote the pion mass and coupling constant, and the up and down quark masses, respectively.

The first step in the direction of solving the strong CP problem with exotic massless quarks was the proposal by K. Choi and E. Kim [11] to enlarge the confining gauge sector of the SM to $S U(3)_{c} \times S U(\tilde{N})$, with the latter having a scale larger than that of QCD, $\tilde{\Lambda} \gg \Lambda_{\mathrm{QCD}}$. Two confined charges would

\footnotetext{
1 The SM $\eta^{\prime}$ is excluded from this definition since the $U(1)_{A}$ symmetry associated to it is broken by the non-zero quark masses.
}

then exist in nature, color and axicolor respectively, and correspondingly two distinct sources of instanton potentials. A massless color-triplet quark $Q$, charged also under axicolor and singlet under the SM electroweak symmetry, would solve the QCD strong CP problem. The fact that the axicolor scale is very large would explain the non-observation of exotic bound states at low energies. An issue arises because there are now two potentially harmful vacuum angles to absorb: $\theta_{c}$ of QCD and $\tilde{\theta}$. Only one combination of them would be redefined away by a chiral rotation of $Q$. This was easily remedied by adding a second exotic quark $\chi$ charged only under $S U(\tilde{N})$. In the limit of vanishing QCD coupling, the $S U(\tilde{N})$ sector described four flavors which seed two singlet pseudoscalars with anomalous couplings: two dynamical axions. Finally, taking into account the SM quark sector and thus the SM $\eta^{\prime}$, three flavor-singlet pseudoscalars result in the low-energy spectrum for only two instanton sources of masses: the $\eta^{\prime}$, a very heavy axion with mass $\sim \tilde{\Lambda}$ and a second axion almost massless and obeying Eq. (2). Because of this last axion, the axicolor construction can be seen as an ultraviolet dynamical completion of the invisible axion paradigm. As usual, a very large $f_{a}$ scale is required to be orders of magnitude larger than the electroweak (EW) one, albeit with the advantage of being free from scalar potential fine-tunings.

In a different and recent attempt [15] to solve the strong CP problem with extra massless quarks, the same $S U(3)_{c} \times S U(\tilde{N})$ confining sector is considered. No light axion remains in the low-energy spectrum, though, as only two pseudoscalar mesons are present which couple to the two anomalous currents: the customary $\eta^{\prime}$ meson and one axion. This is achieved by assuming only one exotic massless quark $Q$ instead of two. In the limit of vanishing QCD coupling, the $S U(\tilde{N})$ sector then describes only three flavors, resulting in only one gauge singlet pseudoscalar with anomalous $S U(\tilde{N})$ couplings: a dynamical axion. Both the $\eta^{\prime}$ and the axion thus acquire a mass, and the axion mass is induced by $S U(\tilde{N})$ instantons, $m_{a}, f_{a} \sim \tilde{\Lambda}$. For such a heavy axion the $f_{a}$ scale can be as low as the TeV range without incurring unacceptable phenomenological consequences [15-24,27]. The issue of the two $\theta$ parameters is solved in this proposal by imposing a discrete $Z_{2}$ symmetry relating the two sectors. Unfortunately, the practical implementation of this idea requires a complete $Z_{2}$-"mirror" copy of the SM. This $Z_{2}$ symmetry is explicitly broken by a scalar potential which gives the second Higgs field a very large vacuum expectation value, e.g. $10^{14} \mathrm{GeV}$, in order to sufficiently modify the running of the two confining scales. This is overall a quite complex, tuned, and large structure.

Even more recently, the SM massless quark avenue has been revisited in a theory that involves a product of $S U(3)$ groups which break spontaneously to QCD [22]. A very interesting aspect developed by the same authors in a previous work [23] is the impact of small size instantons of 
Table 1 The massless fermion sector of the $S U(6)$ construction above the unification scale

\begin{tabular}{llll}
\hline & $S U(6)$ & $S U(2)_{L}$ & $U(1)$ \\
\hline$\Psi_{L}$ & 20 & 1 & 0 \\
\hline
\end{tabular}

the group which undergoes spontaneous symmetry breaking. These instantons are shown to provide a possible extra source of large masses for the putative axions of the model.

We will develop in what follows a new solution to the strong CP problem via massless fermions, in which the issue of the different $\theta$ parameters that arise in the presence of two or more confining groups is solved via color unification. Color unification with massless quarks is attempted here for the first time. This path is an alternative to the axicolortype constructions and will lead to different phenomenology. QCD will be unified with another confining sector singlet under the electroweak gauge symmetry. The color unified theory (CUT) breaks spontaneously to QCD and another confining group. The small-size instantons of the unified color group provide an extra source of high masses for the axions of the theory, and it will be shown that typically no axion remains at low scales. The exotic low-energy spectrum is instead fermionic. Furthermore, it will be shown that interesting new phenomenological signals can be explored at colliders. The complete ultraviolet completion of this idea will be developed, implementing two different scenarios: in one of them the two resulting heavy axions are dynamical, while in the other one axion is elementary.

The structure of the paper can be easily inferred from the Table of Contents.

\section{$2 \mathrm{SU}(6)$ color unification}

We propose a scenario in which QCD is unified with another confining group into $S U(6)$, and a single, strictly massless $S U$ (6) fermion rotates away simultaneously all $\theta$ parameters. The unification path in the context of an extended strong sector to solve the strong CP problem was first proposed by Rubakov long ago [24], in a Grand Unification construction that relied on traditional models à la DFSZ $[25,26]$ with massive exotic fields, and required a $Z_{2}$ mirror copy of the complete SM field content. Another recent attempt [27] using unification ideas also relied on massive exotic fermions $\grave{a}$ la DFSZ. Here we instead consider color unification in the presence of massless fermions. The massless $S U$ (6) fermion belongs to the 20 representation of the $S U$ (6) CUT, having a definite chirality (e.g. left-handed) while being a singlet of the SM $S U(2)_{L} \times U(1)_{Y}$ gauge symmetry, see Table 1 .

At a color unification scale $\Lambda_{\text {CUT }}$ much higher than the $\mathrm{EW}$ one, the $S U$ (6) group breaks into

$$
S U(6) \stackrel{\Lambda_{\mathrm{CUT}}}{\longrightarrow} S U(3)_{c} \times S U(\tilde{3}) \times U(1) \text {. }
$$

Table 2 The massless fermion sector of the $S U$ (6) construction below the unification scale. The notation is such that $\psi_{L}^{c} \equiv\left(\psi^{c}\right)_{L}=\left(\psi_{R}\right)^{c}$

\begin{tabular}{lll}
\hline & $S U(3)_{c}$ & $S U(\tilde{3})$ \\
\hline$\psi_{L}$ & $\square$ & $\bar{\square}$ \\
$\psi_{L}^{c}$ & $\square$ & $\square$ \\
$2 \times \psi_{\nu}$ & 1 & 1 \\
\hline
\end{tabular}

The parameters $\theta_{c}$ of $S U(3)_{c}$ and $\tilde{\theta}$ of $S U(\tilde{3})$ are necessarily equal and unphysical down to the unification scale, and will remain so even below the unification scale as long as $\Psi$ remains massless, protected by chiral symmetry. Under spontaneous symmetry breaking of the CUT symmetry, $\Psi$ decomposes as

$$
\begin{aligned}
\Psi_{L}(20)= & (1,1)(-3)_{L}+(1,1)(+3)_{L}+(3, \overline{3})(-1)_{L} \\
& +(\overline{3}, 3)(+1)_{L},
\end{aligned}
$$

where the charges under the $U$ (1) group in Eq. (3) are shown in parenthesis for completeness. If the components of the $\Psi_{L}$ field are to remain massless under the CUT scale, $S U(\tilde{3})$ must confine. The two confining scales $\Lambda_{\mathrm{QCD}}$ and $\tilde{\Lambda}$ need to be separated with $\tilde{\Lambda} \gg \Lambda_{\mathrm{QCD}}$, as no bound states are observed other than those compatible with QCD. The 20dimensional representation is thus advantageous because all its components charged under QCD are also charged under $S U(\tilde{3})$, and so will form bound states at the higher scale $\tilde{\Lambda}$. This representation is also pseudo-real, and so the theory is anomaly free. The non-trivial issue of how to separate $\tilde{\Lambda}$ and $\Lambda_{\mathrm{QCD}}$ is discussed further below.

The colored-axicolored massless fermions in Eq. (4) will be denoted $\psi_{L, R}$, see Table 2 , while $\psi_{\nu}$ will refer to the singlet massless fermions to convey that they act like sterile $^{2}$ neutrinos. The $\psi_{v}$ fields only connect to the other fields through the unified strong forces, and thus their couplings to the visible universe will be safely suppressed by $\Lambda_{\mathrm{CUT}}$, provided the $U(1)$ gauge group in (3) is also broken near that scale. $^{3}$

$S U$ (6) color unification is thus a successful path to solve the strong $\mathrm{CP}$ problem, and this fact will remain at the heart of the developments in this paper. The remaining problem is to obtain a low-energy spectrum which is fully compatible with observations.

\section{The SM fermions}

Because of color unification, the SM quarks must belong to $S U$ (6) multiplets. The simplest option is to include them in six-dimensional fundamental representations. For each fermion generation,

$$
\begin{aligned}
& Q_{L}(6) \equiv(q, \tilde{q})_{L}, \quad U(6) \equiv(u, \tilde{u})_{R} \\
& D(6) \equiv(d, \tilde{d})_{R}
\end{aligned}
$$

\footnotetext{
${ }^{2}$ By "sterile fermion" is meant any fermion which is not charged under the SM gauge group.

3 This breaking will become manifest in the next section.
} 
where $q_{L}, u_{R}$ and $d_{R}$ denote the SM quarks, while their $S U$ (6) partners are signaled by tildes. The $\tilde{q}_{L}$ fields are necessarily electroweak doublets, and this character turns out to be the major practical issue of this model:

- Leaving the tilde-quark sector massless but confined is unacceptable, as the condensate-assuming chiral symmetry breaking of $S U(\tilde{3})$ - would typically break the SM EW symmetry at the large $\tilde{\Lambda}$ scale.

- Alternatively, giving much larger masses $(\geq \tilde{\Lambda})$ to the tilde quarks is not viable either in this $S U(6)$ setup without spoiling SM quark masses, since they belong to the same multiplet. If a scalar field gave high masses to the tilde quarks ${ }^{4}$ by obtaining a high vacuum expectation value (vev), that scalar field would have to be an $S U(2)_{L}$ doublet. Then its large vev would spontaneously break SM EW symmetry, giving gigantic masses to the $W$ and $Z$ boson.

The main problem of this model is then the unacceptably light tilde-fermion sector. We will develop next an extension whose only purpose is precisely to achieve high masses for the tilde-sector quarks, decoupling them from the low-energy spectrum. By the same token, the necessary separation of $\Lambda_{\mathrm{QCD}}$ and a larger confining scale will naturally follow. ${ }^{5} \mathrm{We}$ will develop in detail two realistic ultraviolet (UV) completions.

\section{The realistic color unified theory: $S U(6) \times S U\left(3^{\prime}\right)$}

It is necessary to give large masses to the tilde-quark sector without giving masses to the SM quarks, a challenging enterprise as explained above due to the $S U(6)$ unification. An external mechanism is ideal for this task. The color unified $S U(6)$ group which contains QCD is enlarged via an external non-abelian $S U\left(3^{\prime}\right)$ group with additional fermions charged only under the latter. In fact, all fermions in the theory will be charged under only one of the two groups, $S U$ (6) or $S U\left(3^{\prime}\right) . \Psi_{L}$ will be thus taken to be a singlet of $S U\left(3^{\prime}\right)$ and the same applies to the multiplets in Eq. (5) which contain the SM quarks. The two sectors are connected exclusively via a new scalar $\Delta$. QCD remains a subgroup of $S U(6)$, whose $\theta$-parameter is rotated away by the massless $\Psi_{L}$ fermion in Eq. (4). This type of auxiliary extension was suggested in

\footnotetext{
4 Through tuned Yukawa couplings of the tilde-quark sector to an extended scalar sector.

${ }^{5}$ If $S U$ (6) sufficed to obtain a realistic spectrum, the $S U(\tilde{3})$ group and $\tilde{\Lambda}$ scale of this section would correspond to those of the axicolor group [11] as described in the introduction. The extension of the CUT group will break this direct correspondence, although two confining groups will still be at play.
}

Ref. [27] to give high masses to exotic fermions in a different context. The field content of our model is summarized in Table 3, in which all fermions except $\Psi_{L}$ will become massive. It is easy to see that the theory with this matter content is anomaly free. The scalar $\Delta$ appearing in the table belongs to the bifundamental of $S U(6) \times S U\left(3^{\prime}\right)$, and its vev breaks color unification at a scale $\Lambda_{\mathrm{CUT}}$, taken to be much larger than all SM scales,

$S U(6) \times S U\left(3^{\prime}\right) \stackrel{\Lambda_{\mathrm{CUT}}}{\longrightarrow} S U(3)_{c} \times S U(3)_{\mathrm{diag}}$.

The fermion quantum numbers under the two resulting groups are also shown in Table 3.

A simple CUT-invariant Yukawa Lagrangian which connects the $S U(6)$ and the auxiliary $S U\left(3^{\prime}\right)$ extension reads

$\mathcal{L} \ni \kappa_{q} q_{L}^{\prime} \Delta^{*} Q_{L}+\kappa_{u} u_{L}^{\prime c} \Delta U_{L}^{c}+\kappa_{d} d_{L}^{\prime c} \Delta D_{L}^{c}+$ h.c..

The CUT symmetry is spontaneously broken upon $\Delta$ taking a vev of the order of the CUT breaking scale $\Lambda_{\text {CUT }}$

$\langle\Delta\rangle=\Lambda_{\text {CUT }}\left(\begin{array}{llllll}0 & 0 & 0 & 1 & 0 & 0 \\ 0 & 0 & 0 & 0 & 1 & 0 \\ 0 & 0 & 0 & 0 & 0 & 1\end{array}\right)$.

This breaking generates a large mass for both the tilde- and prime-quark sectors, leaving massless only the SM fermion components of the original fermionic fields: ${ }^{6}$

$\mathcal{L} \ni \Lambda_{\mathrm{CUT}}\left\{\kappa_{q} q_{L}^{\prime} \tilde{q}_{L}+\kappa_{u} u_{L}^{\prime c} \tilde{u}_{L}^{c}+\kappa_{d} d_{L}^{\prime c} \tilde{d}_{L}^{c}\right\}+$ h.c.

Unless otherwise stated, we will assume in what follows that all $\kappa_{i}$ Yukawa couplings are $\mathcal{O}(1)$, meaning all tilde and prime fermion masses are of order $\Lambda_{\mathrm{CUT}}$. Some tuning of the $\kappa_{i}$ values could be acceptable, though, as discussed further below.

The SM fermions get their masses through the usual SM Higgs doublet $\Phi$, which in this model is a singlet of $S U(6) \times$ $S U\left(3^{\prime}\right),{ }^{7}$

$\mathcal{L} \ni Y_{u}^{S M} Q_{L} \Phi U_{L}^{c}+Y_{d}^{S M} Q_{L} \tilde{\Phi} D_{L}^{c}+$ h.c.,

where $Y_{i}^{S M}$ denote the SM Yukawa couplings. Analogously, the most general Lagrangian compatible with all symmetries discussed above allows us to write Yukawa couplings of the Higgs field to the prime-sector fermions,

$\mathcal{L} \ni y_{u}^{\prime} q_{L}^{\prime} \Phi u_{L}^{\prime c}+y_{d}^{\prime} q_{L}^{\prime} \tilde{\Phi} d_{L}^{\prime c}+$ h.c. .

\footnotetext{
${ }^{6}$ Note that we take $\langle\Delta\rangle$ to be real. The phases of the nonvanishing entries in Eq. (8) can all be made equal by an $S U(6) \times S U\left(3^{\prime}\right)$ transformation; the remaining phase can be removed by a transformation under the $U(1)$ defined in Eq. (12).

${ }^{7}$ In this notation taken from unified models the contraction of the spinor indices is implicit, more precisely the first term would read $Q_{L}^{T} C \Phi U_{L}^{c}$, where $C=i \gamma_{2} \gamma_{0}$ is the charge conjugation matrix.
} 
Table 3 The matter content. The table on the left describes matter above the CUT scale, while the one on the right gives the transformation properties under the gauge groups remaining after CUT spontaneous breaking. The fermions in bold have masses comparable to $\Lambda_{\mathrm{CUT}}$ and are integrated out around the CUT scale. The quantum numbers under the EW gauge group correspond both to the high-energy and low-energy fields

\begin{tabular}{llllllll}
\hline & $S U(6)$ & $S U\left(3^{\prime}\right)$ & & $S U(3)_{c}$ & $S U(3)_{\text {diag }}$ & $S U(2)_{L}$ & $U(1)_{Y}$ \\
\hline$Q_{L}$ & $\square$ & 1 & $q_{L}$ & $\square$ & 1 & $\square$ & $\frac{1}{6}$ \\
& & & $\tilde{\mathbf{q}}_{\mathbf{L}}$ & 1 & $\square$ & $\square$ & $\frac{1}{6}$ \\
$U_{L}^{c}$ & $\square$ & 1 & $u_{L}^{c}$ & $\square$ & 1 & 1 & $-\frac{2}{3}$ \\
& & & $\tilde{\mathbf{u}}_{\mathbf{L}}^{\mathbf{c}}$ & 1 & $\square$ & 1 & $-\frac{2}{3}$ \\
$D_{L}^{c}$ & $\square$ & 1 & $d_{L}^{c}$ & $\square$ & 1 & 1 & $\frac{1}{3}$ \\
& & & $\tilde{\mathbf{d}}_{\mathbf{L}}^{\mathbf{c}}$ & 1 & $\square$ & 1 & $\frac{1}{3}$ \\
$\Psi_{L}$ & 20 & 1 & $\psi_{L}$ & $\square$ & $\square$ & 1 & 0 \\
& & & $\psi_{L}^{c}$ & $\square$ & $\square$ & 1 & 0 \\
& & & $2 \times \psi_{\nu}$ & 1 & 1 & 1 & 1 \\
$q_{L}^{\prime}$ & 1 & $\square$ & $\mathbf{q}_{\mathbf{L}}^{\prime}$ & 1 & $\square$ & $\square$ & $-\frac{1}{6}$ \\
$u_{L}^{\prime c}$ & 1 & $\square$ & $\mathbf{u}_{\mathbf{L}}^{\mathbf{c}}$ & 1 & $\square$ & 1 & $\frac{2}{3}$ \\
$d_{L}^{\prime c}$ & 1 & $\square$ & $\mathbf{d}_{\mathbf{L}}^{\mathbf{c}}$ & 1 & $\square$ & 1 & $-\frac{1}{3}$ \\
$\Delta$ & $\square$ & $\square$ & - & - & - & 1 & 0 \\
\hline
\end{tabular}

Eqs. (10) and (11) induce contributions to the tilde fermion masses which are quantitatively irrelevant in comparison with those from Eq. (9). In addition, the couplings in Eq. (11) will be absent for symmetry reasons in one of the models to be developed in this paper (Model II in Sect. 3.2).

Both $S U(3)_{c}$ and $S U(3)_{\text {diag }}$ can now remain unbroken and confine at two different scales, $\Lambda_{\mathrm{QCD}}$ and $\Lambda_{\text {diag }}$, with $\Lambda_{\text {diag }} \gg \Lambda_{\mathrm{QCD}}$. The task of achieving different values for the two confining scales and getting rid of the tilde sector or any other dangerous exotic sector is thus accomplished.

Note that the Yukawa-type Lagrangian in Eq. (7) has an inherent global $U(1)$ symmetry under which only the prime fermions and the $\Delta$ field would transform - a generalized Baryon number symmetry in the prime sector, with charges

$B N^{\prime}\left\{\Delta, q_{L}^{\prime}, u_{L}^{\prime c}, d_{L}^{\prime c}\right\}=\{+1,+1,-1,-1\}$.

This symmetry is not chiral, thus not anomalous under $S U\left(3^{\prime}\right)$, and irrelevant to the strong CP problem. An associated $\mathrm{pGB}^{8}$ results after spontaneous breaking, albeit with its couplings safely suppressed by the CUT scale and interacting only with the very heavy prime and tilde sectors. ${ }^{9}$

Finally, the theory below $\Lambda_{\mathrm{CUT}}$ contains phenomenologically interesting bound states formed from the massless $\psi_{L, R}$ fermions, to be studied below. The spectrum of free eigenstates below the EW scale contains the usual SM spectrum, plus a harmless pGB and sterile neutrinos.

\footnotetext{
8 This symmetry is broken at loop level by $S U(2)_{L}$ sphalerons, in the same way that in the SM baryon number current is anomalous. For our purposes this effect is negligible.

9 As suggested in Ref. [23], this type of pGB could be entirely removed by gauging the $\mathrm{U}(1)$ group. There is no real need to implement this procedure in our case, though, given the strongly suppressed couplings of this pGB.
}

\section{$\theta^{\prime}$ issue}

The extension of the strong sector by the auxiliary external group $S U\left(3^{\prime}\right)$ brings a new $\theta^{\prime}$ parameter into the game:

$$
\begin{aligned}
\mathcal{L} \supset \theta_{6} \frac{\alpha_{6}}{8 \pi} G_{6} \tilde{G}_{6}+\theta^{\prime} \frac{\alpha^{\prime}}{8 \pi} G^{\prime} \tilde{G}^{\prime} \\
\longrightarrow\left(\theta_{6}+\theta^{\prime}\right) \frac{\alpha_{\mathrm{diag}}}{8 \pi} G_{\mathrm{diag}} \tilde{G}_{\mathrm{diag}}+\theta_{6} \frac{\alpha_{c}}{8 \pi} G_{c} \tilde{G}_{c},
\end{aligned}
$$

where $G_{i}$ denote gauge field strengths with tensorial indices omitted. $G_{c}, G_{6}, G^{\prime}$ and $G_{\text {diag }}$ correspond respectively to the SM QCD gauge group, $S U(6), S U\left(3^{\prime}\right)$ and $S U(3)_{\text {diag. While }}$ the rotation of the massless field $\Psi$ was designed to reabsorb $\theta_{6}$ and ultimately $\theta_{c}, \theta^{\prime}$ may source back a SM strong CP problem through the contamination to the visible sector via the $\Delta$ scalar.

Indeed, at low energies the massless quark $\psi$ transforms as a $(3, \overline{3})$, therefore the phase $\theta_{6}$ cannot be fully reabsorbed in the Lagrangian since the chiral rotation that removes the $S U(3)_{c} \theta$-term generates a new contribution to the $S U(3)_{\text {diag }}$ topological term. Reference [27] acknowledges this issue (in the context of a different model which does not rely on massless fermions) and leaves it unsolved hoping that some UV completion solves it. In what follows, we will determine and exhaustively analyze two UV solutions, via the simple addition of either

- An extra massless fermion transforming only under $S U\left(3^{\prime}\right)$.

- A second bifundamental scalar field, which automatically endows PQ invariance to the above extension procedure.

The first solution is more in line with the spirit of the present paper, as all $\theta$ parameters inducing a strong $\mathrm{CP}$ problem are 
Table 4 The table shows on the left (right) the quantum numbers above (under) the CUT scale for the massless $\chi$ quarks which absorbs $\theta^{\prime}$ in Model I

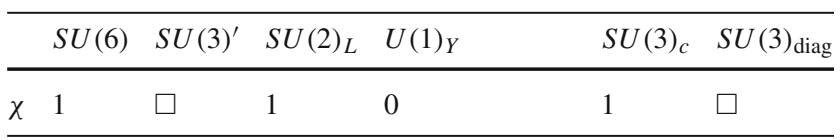

made unphysical via massless fermions, and it is developed next.

3.1 Model I: adding a massless fermion charged under $S U\left(3^{\prime}\right)$

The $\theta^{\prime}$ parameter of the auxiliary $S U\left(3^{\prime}\right)$ gauge group can be made unphysical by the addition of a massless fermion field $\chi$ that transforms as a fundamental of $S U\left(3^{\prime}\right)$ and is an EW and $S U(6)$ singlet. In other words, the field content for this solution is that previously shown in Table 3 plus the massless fermion $\chi$ with quantum numbers shown in Table 4. Additional composite bound states will result from $\chi$, among them composite pseudoscalars with anomalous couplings - dynamical axions - whose masses are discussed further below.

\subsubsection{Running of the coupling constants}

The CUT breaking pattern in Eq. (6) imposes the following relations among the gauge couplings

$\frac{1}{\alpha_{\mathrm{diag}}(\mu)}=\frac{1}{\alpha_{6}(\mu)}+\frac{1}{\alpha^{\prime}(\mu)}, \quad$ at $\quad \mu=\Lambda_{\mathrm{CUT}}$,

with the constraint

$\alpha_{c}\left(\Lambda_{\mathrm{CUT}}\right)=\alpha_{6}\left(\Lambda_{\mathrm{CUT}}\right)$

where $\alpha_{c}, \alpha_{\text {diag }}, \alpha^{\prime}$ and $\alpha_{6}$ denote respectively the coupling strength of QCD, $S U(3)_{\mathrm{diag}}, S U\left(3^{\prime}\right)$ and $S U(6)$. As shown in Fig. 1, there is a discontinuity in the running of the coupling constants at the CUT-breaking scale that allows $\alpha^{\prime}$ to have large values while reproducing the known QCD running at low scales. ${ }^{10}$ Those $\alpha^{\prime}$ values will seed a source of large axion masses, as discussed in Sect. 3.1.3 further below.

Although the relation in Eq. (14) imposes $\alpha_{\text {diag }}\left(\Lambda_{\mathrm{CUT}}\right)<$ $\alpha_{c}\left(\Lambda_{\mathrm{CUT}}\right)$, the presence of the SM $q_{L}, u_{R}$, and $d_{R}$ quarks at energies well below $\Lambda_{\text {CUT }}$ slows down the running of QCD

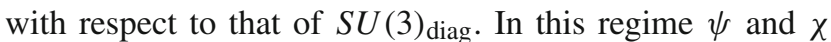
are the only fields left charged under $S U(3)_{\text {diag }}$ [the $\tilde{q}$ sector

\footnotetext{
10 Figure 1 assumes a zero $\chi$ mass. As will be shown in Sect. 3.1.5, $\chi$ acquires an effective mass due to small-size instanton effects. Threshold effects near $m_{\chi}$ may alter the running. Even when these effects are large enough to be noticeable, Fig. 1 still captures the qualitative behavior of the RG flow.
}

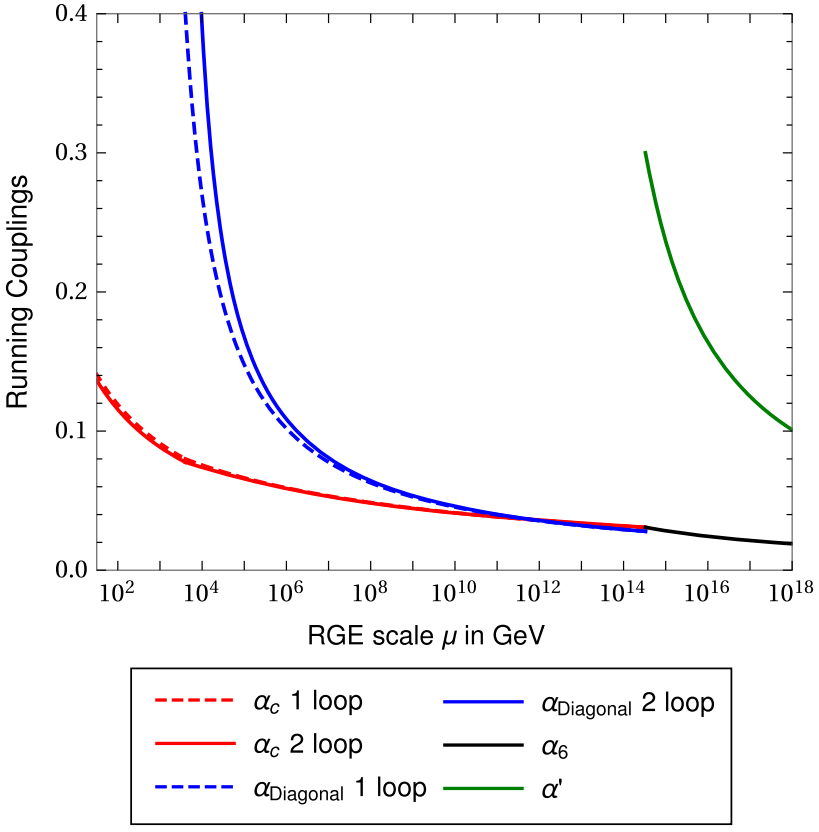

Fig. 1 Running of $\alpha_{\mathrm{QCD}}, \alpha_{\text {diag }}, \alpha_{6}$, and $\alpha^{\prime}$ in the model with the one extra $S U\left(3^{\prime}\right)$ fermion of Table 4 . The full matter content is given by both Tables 3 and 4 . The inputs used are $\alpha^{\prime}\left(\Lambda_{\mathrm{CUT}}\right)=0.3$ and $\Lambda_{\mathrm{CUT}}=$ $3.3 \times 10^{14} \mathrm{GeV}$ for illustration, which results in $\Lambda_{\text {diag }}=4 \mathrm{TeV}$ (taken as a benchmark point). The solid (dashed) lines correspond to the two (one) loop results

Table 5 The massless quark sector charged under $S U(3)_{\text {diag }}$ remaining below the confining scale $\Lambda_{\text {diag }}$

generically decouples as their mass scale is set by $\Lambda_{\mathrm{CUT}}$, see Eq. (9)]. As a consequence, $\alpha_{\text {diag }}$ runs faster and thus the $S U(3)_{\text {diag }}$ group confines at a higher scale than $\Lambda_{\mathrm{QCD}}$, see Fig. 1. This mechanism easily achieves the separation of the two confining scales. We computed both the one- and twoloop running and the latter actually reinforces the pattern, as illustrated in the figure for the choice $\Lambda_{\text {diag }}=4 \mathrm{TeV}$. Lower values of $\Lambda_{\text {diag }}$ are also phenomenologically acceptable, see Sect. 4.1 below.

\subsubsection{Confinement of $S U(3)_{\mathrm{diag}}$ and pseudoscalar anomalous couplings to the confining interactions}

At the scale $\Lambda_{\text {diag }}, S U(3)_{\text {diag }}$ confines and the remaining massless fermions will form massive QCD-colored bound states.

Assuming that both $\psi$ and $\chi$ in Table 5 remain massless after $S U(3)_{\text {diag }}$ confinement, the $S U(3)_{\text {diag Lagrangian }}$ exhibits, at the classical level and in the limit in which $\alpha_{c}$ is 
switched off, a global flavor symmetry $U(4)_{L} \times U(4)_{R} \longrightarrow$ $U(4)_{V} \cdot{ }^{11}$ The chiral symmetry is then spontaneously broken by the quark condensates $\left\langle\bar{\psi}_{L} \psi_{R}\right\rangle$ and $\left\langle\bar{\chi}_{L} \chi_{R}\right\rangle$. This results in $16(\mathrm{p}) \mathrm{GBs}$,

$16=8_{c}+\overline{3}_{c}+3_{c}+1_{c}+1_{c}$,

decomposed here in terms of their QCD charges. There is a QCD octet plus a singlet with flavor content $\bar{\psi} \psi\left(\overline{3}_{c} \times 3_{c}=\right.$ $8_{c}+1_{c}$ ). The two QCD triplets, $3_{c}$ and $\overline{3}_{c}$, correspond to the combinations $\bar{\psi} \chi$ and $\bar{\chi} \psi$. Finally, a color-singlet composite state is made out of $\bar{\chi} \chi$. The fourteen colored mesons in Eq. (16) acquire large masses induced by gluon loops that are quadratically divergent and therefore sensitive to the cutoff scale $\Lambda_{\text {diag }},{ }^{12}$

$m^{2}\left(8_{c}\right) \approx \frac{9 \alpha_{\mathrm{c}}}{4 \pi} \Lambda_{\mathrm{diag}}^{2}, \quad m^{2}\left(\overline{3}_{c}\right) \approx m^{2}\left(3_{c}\right) \approx \frac{\alpha_{\mathrm{c}}}{\pi} \Lambda_{\mathrm{diag}}^{2}$

The remaining two QCD singlets will be denoted here by $\eta_{\psi}^{\prime}$ and $\eta_{\chi}^{\prime}$ and are shown next to be dynamical axions. The associated currents are

$j_{\psi_{A}}^{\mu}=\bar{\psi} \gamma^{\mu} \gamma^{5} t^{9} \psi \equiv f_{\mathrm{d}} \partial^{\mu} \eta_{\psi}^{\prime}, \quad t^{9}=\frac{1}{\sqrt{6}} \mathbb{1}_{3 \times 3}$,

$j_{\chi_{A}}^{\mu}=\bar{\chi} \gamma^{\mu} \gamma^{5} \chi \equiv f_{\mathrm{d}} \partial^{\mu} \eta_{\chi}^{\prime}$,

where $f_{\mathrm{d}}$ denotes the $S U(3)_{\text {diag }}$ pGB scale, with $\Lambda_{\text {diag }} \leq$ $4 \pi f_{\mathrm{d}}$. These classically conserved currents are broken at the quantum level by the $S U(6)$ and $S U\left(3^{\prime}\right)$ instantons, and so the currents are anomalous. The anomalous terms are

$$
\begin{aligned}
\partial_{\mu} j_{\psi_{A}}^{\mu} & =-\sqrt{6} \frac{\alpha_{6}}{8 \pi} G_{6} \tilde{G}_{6} \\
& \longrightarrow-\sqrt{6} \frac{\alpha_{\mathrm{diag}}}{8 \pi} G_{\mathrm{diag}} \tilde{G}_{\mathrm{diag}}-\sqrt{6} \frac{\alpha_{c}}{8 \pi} G_{c} \tilde{G}_{c} \\
\partial_{\mu} j_{\chi_{A}}^{\mu} & =-2 \frac{\alpha^{\prime}}{8 \pi} G^{\prime} \tilde{G}^{\prime} \longrightarrow-2 \frac{\alpha_{\mathrm{diag}}}{8 \pi} G_{\mathrm{diag}} \tilde{G}_{\mathrm{diag}} .
\end{aligned}
$$

These anomalous terms modify the classical equations of motion of the $\eta_{\psi}^{\prime}$ and $\eta_{\chi}^{\prime}$,

$$
\begin{array}{r}
f_{\mathrm{d}} \square \eta_{\psi}^{\prime}=-\sqrt{6} \frac{\alpha_{6}}{8 \pi} G_{6} \tilde{G}_{6}, \\
f_{\mathrm{d}} \square \eta_{\chi}^{\prime}=-2 \frac{\alpha^{\prime}}{8 \pi} G^{\prime} \tilde{G}^{\prime},
\end{array}
$$

$11 U(4)_{V}$ remains unbroken and contains as a subgroup the $S U(3)_{c}$ QCD gauge group.

12 They contribute to the running of the QCD coupling constant, but given their high masses their impact is unnoticeable. and give rise to an effective Lagrangian,

$$
\begin{aligned}
\mathcal{L} \supset & -\frac{\alpha_{6}}{8 \pi} \frac{\sqrt{6} \eta_{\psi}^{\prime}}{f_{\mathrm{d}}} G_{6} \tilde{G}_{6}-\frac{\alpha^{\prime}}{8 \pi} \frac{2 \eta_{\chi}^{\prime}}{f_{\mathrm{d}}} G^{\prime} \tilde{G}^{\prime} \\
\longrightarrow & -\frac{\alpha_{c}}{8 \pi} \frac{\sqrt{6} \eta_{\psi}^{\prime}}{f_{\mathrm{d}}} G_{c} \tilde{G}_{c} \\
& -\frac{\alpha_{\text {diag }}}{8 \pi}\left(2 \frac{\eta_{\chi}^{\prime}}{f_{\mathrm{d}}}+\sqrt{6} \frac{\eta_{\psi}^{\prime}}{f_{\mathrm{d}}}\right) G_{\text {diag }} \tilde{G}_{\text {diag }} .
\end{aligned}
$$

$\eta_{\psi}^{\prime}$ and $\eta_{\chi}^{\prime}$ are thus two dynamical axions. It is to be stressed that the PQ scale in this model is $f_{\mathrm{d}} \sim \Lambda_{\text {diag }}$ for both axions and not the much larger $\Lambda_{\text {CUT }}$ scale. When the SM quarks are taken into account, the $\eta_{\mathrm{QCD}}^{\prime}$ pseudoscalar meson is also present at energies below the QCD confinement scale, and the effective Lagrangian of anomalous couplings reads

$$
\begin{gathered}
\mathcal{L} \supset-\frac{\alpha_{\mathrm{diag}}}{8 \pi}\left(2 \frac{\eta_{\chi}^{\prime}}{f_{\mathrm{d}}}+\sqrt{6} \frac{\eta_{\psi}^{\prime}}{f_{\mathrm{d}}}\right) G_{\mathrm{diag}} \tilde{G}_{\mathrm{diag}} \\
-\frac{\alpha_{c}}{8 \pi}\left(2 \frac{\eta_{\mathrm{QCD}}^{\prime}}{f_{\pi}}+\sqrt{6} \frac{\eta_{\psi}^{\prime}}{f_{\mathrm{d}}}\right) G_{c} \tilde{G}_{c},
\end{gathered}
$$

where $\Lambda_{\mathrm{QCD}} \leq 4 \pi f_{\pi}$.

$$
\begin{aligned}
V_{e f f}= & \frac{\mathcal{E}_{\text {diag }}^{4}}{2}\left(2 \frac{\eta_{\chi}^{\prime}}{f_{\mathrm{d}}}+\sqrt{6} \frac{\eta_{\psi}^{\prime}}{f_{\mathrm{d}}}\right)^{2} \\
& +\frac{\mathcal{E}_{\mathrm{QCD}}^{4}}{2}\left(2 \frac{\eta_{\mathrm{QCD}}^{\prime}}{f_{\pi}}+\sqrt{6} \frac{\eta_{\psi}^{\prime}}{f_{\mathrm{d}}}\right)^{2} .
\end{aligned}
$$

The scale $\mathcal{E}_{\mathrm{QCD}}$ can be expressed in terms of QCD observables from the chiral effective Lagrangian according to the results from Refs. [28-30] obtained in the large $N$ limit. In the two-quark approximation, $\mathcal{E}_{\mathrm{QCD}}$ reads

$\mathcal{E}_{\mathrm{QCD}}^{4}=\frac{f_{\eta}^{2} m_{\eta}^{2}-f_{\pi}^{2} m_{\pi}^{2}}{4} \simeq(202 \mathrm{MeV})^{4} \simeq \Lambda_{\mathrm{QCD}}^{4}$,

and $\mathcal{E}_{\text {diag }}$ is obtained by rescaling the previous value

$\mathcal{E}_{\text {diag }}^{4}=\mathcal{E}_{\mathrm{QCD}}^{4}\left(\frac{\Lambda_{\text {diag }}}{\Lambda_{\mathrm{QCD}}}\right)^{4} \simeq \Lambda_{\text {diag }}^{4}$.

As a consequence, the two instanton-induced scales $\Lambda_{\mathrm{QCD}}$ and $\Lambda_{\text {diag }}$ provide a contribution to the masses of the pseudoscalars which have anomalous couplings.

It follows that there are only two sources of mass (disregarding corrections from SM quark masses) for three states coupling to anomalous currents: $\eta_{\mathrm{QCD}}^{\prime}, \eta_{\psi}^{\prime}$ and $\eta_{\chi}^{\prime}$. In the absence of supplementary mass sources, one axion would get a mass of order $\Lambda_{\text {diag }}$ while another one would have remained almost massless, see Eqs. (1) and (2), as often happens in models with dynamical axions. The model would be simply an ultraviolet implementation of the invisible axion paradigm. 
As we will see next, an additional and important instanton source of mass for the axions is present, though, which lifts the light axion mass. In fact, depending on the model parameters, the $\chi$ fermion mass itself may: (1) still be lighter than $\Lambda_{\text {diag }}$, in which case the above discussed $U$ (4) pattern of global symmetry holds; (2) alternatively, become more massive than $\Lambda_{\text {diag }}$ and thus be decoupled from the spectrum above $\Lambda_{\text {diag. }}$. In the latter case, the approximate global flavor symmetry of the $S U(3)_{\text {diag }}$ Lagrangian would instead be $U(3)_{L} \times U(3)_{R} \longrightarrow U(3)_{V}$, suggesting $9(\mathrm{p}) \mathrm{GBs}$,

$9=8_{c}+1_{c}$.

The mass of this QCD-colored octet pseudoscalar is as previously given in Eq. (17) above, while the $\eta_{\chi}^{\prime}$ would then disappear from the spectrum at energies above $\Lambda_{\text {diag }}$ : the presence of $\eta_{\chi}^{\prime}$ is to be then disregarded in Eqs. (19)-(26).

\subsubsection{The impact of small-size instantons on the dynamical axion mass}

An additional and putatively large contribution to the axion mass(es) applies in the presence of a spontaneously broken theory: the small-size instantons (SSI) of the theory at the breaking scale, as pointed out long ago in Refs. [31-33] and very recently in Ref. [23]. SSI can induce a large mass even for perturbative theories if the breaking scale is large enough to overcome the exponential suppression of instanton effects. In our model, the instantons of the color-unified theory in Eq. (6) near the $\Lambda_{\mathrm{CUT}}$ scale provide automatically this third source of axion mass. The $S U$ (6) SSI can be neglected because of the smallness of $\alpha_{6}$ at $\Lambda_{\mathrm{CUT}}$ (e.g. see Fig. 1) and the analysis below will focus on the $S U\left(3^{\prime}\right)$ SSI contribution.

At the scales where we will compute the SSI effects, the $S U\left(3^{\prime}\right)$ gauge coupling is perturbative. Therefore, for these instantons, the dilute gas approximation [34] gives a reliable estimate of the effective potential for the pseudoscalars. This was not the case for the previous instanton effects which, corresponding to the confinement scales, required the use of the effective chiral Lagrangian to obtain the potential from QCD observables.

It is well known [34-37] that, in the absence of fermions, the effective Lagrangian that describes instanton configurations for a pure Yang-Mills theory $S U\left(N_{c}\right)$ induces a scale $\Lambda_{\text {inst }}$ in the instanton potential given by

$\Lambda_{\text {inst }}^{4}=\int \frac{d \rho}{\rho^{5}} D\left[\alpha^{\prime}(1 / \rho)\right]$,

where $\rho$ is the instanton size, $D\left[\alpha^{\prime}\right]$ is the dimensionless instanton density,

$D\left[\alpha^{\prime}(1 / \rho)\right]=C_{\text {inst }}\left(\frac{2 \pi}{\alpha^{\prime}(1 / \rho)}\right)^{2 N_{c}} e^{-2 \pi / \alpha^{\prime}(1 / \rho)}$.
The constant $C_{\text {inst }}$ reads [38,39]

$C_{i n s t}\left(N_{c}\right)=\frac{4}{\pi^{2}} \frac{2^{-2 N_{c}} e^{-c(1)-2\left(N_{c}-2\right) c(1 / 2)}}{\left(N_{c}-2\right) !\left(N_{c}-1\right) !}$,

and the function $c(x)$ is defined in Ref. [35] such that $c(1 / 2)=$ 0.145873 and $c(1)=0.443307$. For the $S U\left(3^{\prime}\right)$ instantons of our model $C_{\text {inst }}=0.0015 .{ }^{13}$ In order to compute the integral in Eq. (30), the running of the coupling constant $\alpha^{\prime}(\mu)$ must be included. At one loop this reads

$\frac{2 \pi}{\alpha^{\prime}(\mu)}=b \ln \left(\mu / \Lambda_{\mathrm{CUT}}\right)+\frac{2 \pi}{\alpha_{\mathrm{CUT}}^{\prime}}$,

where $\alpha_{\mathrm{CUT}}^{\prime} \equiv \alpha^{\prime}\left(\Lambda_{\mathrm{CUT}}\right)$ and $b$ is the one-loop $\beta$-function coefficient. For the spontaneously broken theory, only the SSI instantons with size $\leq 1 / \Lambda_{\text {CUT }}$ are relevant [40],

$$
\begin{aligned}
\Lambda_{\mathrm{SSI}}^{4}= & C_{i n s t} e^{-2 \pi / \alpha_{\mathrm{CUT}}^{\prime}} \int_{0}^{1 / \Lambda_{\mathrm{CUT}}} \frac{d \rho}{\rho^{5}}\left(\rho \Lambda_{\mathrm{CUT}}\right)^{b} \\
& \left(-b \ln \left(\rho \Lambda_{\mathrm{CUT}}\right)+\frac{2 \pi}{\alpha^{\prime}\left(\Lambda_{\mathrm{CUT}}\right)}\right)^{6} .
\end{aligned}
$$

This has the form

$\Lambda_{\mathrm{SSI}}^{4}=C_{\text {inst }} f\left(\alpha_{\mathrm{CUT}}^{\prime}, b\right) e^{-\frac{2 \pi}{\alpha_{\mathrm{CUT}}^{\prime}}} \Lambda_{\mathrm{CUT}}^{4}$,

where $f\left(\alpha^{\prime}, b\right)$ is given by

$$
\begin{aligned}
f\left(\alpha^{\prime}, b\right)= & \frac{16}{\alpha^{6}(b-4)^{7}} \\
& \left(45 \alpha^{\prime 6} b^{6}+90 \pi \alpha^{15} b^{5}(b-4)\right. \\
& +90 \pi^{2} \alpha^{\prime 4} b^{4}(b-4)^{2}+60 \pi^{3} \alpha^{3} b^{3}(b-4)^{3} \\
& +30 \pi^{4} \alpha^{\prime 2} b^{2}(b-4)^{4}+12 \pi^{5} \alpha^{\prime} b(b-4)^{5} \\
& \left.+4 \pi^{6}(b-4)^{6}\right) .
\end{aligned}
$$

For instance for the benchmark value $\alpha_{\mathrm{CUT}}^{\prime}=0.3$, the value of $S U\left(3^{\prime}\right)$ SSI-induced scale in the absence of fermions (for which $b=10)$ is

$\Lambda_{\mathrm{SSI}}^{4}=3.0 \times 10^{-5} \Lambda_{\mathrm{CUT}}^{4}$

Nevertheless, the presence of fermions dramatically changes the value of this scale [36]. A suppression factor appears, which results from the interplay of the instantons of the theory and the fermionic spectrum. For the $S U\left(3^{\prime}\right)$ theory under consideration, the prime-fermion Yukawa couplings are relevant.

\footnotetext{
${ }^{13}$ This value differs from that used in Ref. [23] $\left(C_{\text {inst }}=0.1\right)$ that was taken directly from the original 't Hooft's computation in Ref. [35], for which it was later shown [38] that the factor $2^{-2 N_{c}}$ was missing. See also Erratum in Ref. [35].
} 


\subsubsection{Small-size instantons with small Yukawa couplings}

The massless $\chi$ fermions may acquire an effective mass due to the instantons of $S U(3)_{\text {diag }}$ and $S U\left(3^{\prime}\right)$ SSI, similar to the effective mass in QCD for a hypothetically massless SM up quark. In this section, we assume the $y_{i}^{\prime}$ couplings in Eq. (11) to be small, e.g $\mathcal{O}(0.2)$ for illustration. This is a regime of moderate small-size instanton effects, in which the instantoninduced effective $\chi$ mass is smaller than the confinement scale. As a consequence, a composite axion made out of this fermion can be considered to be a pGB. ${ }^{14}$

The impact of the $y_{i}^{\prime}$ Yukawa couplings is illustrated by the one-instanton "flower" contribution in the left side of Fig. 2. The pure gauge results in Eq. (30) are now suppressed by two factors: the $\chi$ chiral condensate and the $y_{i}^{\prime}$ dependence,

$$
\begin{aligned}
\Lambda_{\mathrm{SSI}}^{4}= & -\int \frac{d \rho}{\rho^{5}} D\left[\alpha^{\prime}(1 / \rho)\right]\left(\frac{2}{3} \pi^{2} \rho^{3}\langle\bar{\chi} \chi\rangle\right) \\
& \frac{1}{(4 \pi)^{6}} \prod_{i} y_{u}^{\prime i} y_{d}^{\prime i} .
\end{aligned}
$$

$\langle\bar{\chi} \chi\rangle$ is the order parameter controlling $S U(3)_{\text {diag }}$ chiral symmetry breaking and thus expected to be $\langle\bar{\chi} \chi\rangle \simeq-\Lambda_{\text {diag }}^{3}$. For $\rho \leq 1 / \Lambda_{\mathrm{CUT}}$, the product $\rho^{3}\langle\bar{\chi} \chi\rangle \ll 1$ reduces the SSI-induced scale by orders of magnitude, with

$$
\begin{aligned}
\Lambda_{\mathrm{SSI}}^{4}= & \frac{2 \pi^{2}}{3} C_{\text {inst }} \Lambda_{\mathrm{CUT}}^{b} \Lambda_{d}^{3} e^{-2 \pi / \alpha_{\mathrm{CUT}}^{\prime}} \frac{1}{(4 \pi)^{6}} \prod_{i} y_{u}^{\prime i} y_{d}^{\prime i} \\
& \int_{0}^{1 / \Lambda_{\mathrm{CUT}}} d \rho \rho^{b-2}\left(-b \ln \left(\rho \Lambda_{\mathrm{CUT}}\right)+\frac{2 \pi}{\alpha_{\mathrm{CUT}}^{\prime}}\right)^{6}
\end{aligned}
$$

where, in the presence of $N_{f}$ Dirac fermions,

$$
C_{\text {inst }}\left(N_{f}, N_{c}\right)=\frac{4 \cdot 2^{-2 N_{c}} e^{c_{1 / 2}\left(-2 N_{c}+2 N_{f}\right)}}{\pi^{2}\left(N_{c}-2\right) !\left(N_{c}-1\right) !} e^{-c_{1}+4 c_{1 / 2}} .
$$

The integral in Eq. (39) can be computed exactly, although a good estimation follows from the approximation

$$
\begin{aligned}
& \left(1+\frac{-b \alpha_{\mathrm{CUT}}^{\prime}}{2 \pi} \ln \left(\rho \Lambda_{\mathrm{CUT}}\right)\right)^{6} \\
& \simeq 1+6 \frac{-b \alpha_{\mathrm{CUT}}^{\prime}}{2 \pi} \ln \left(\rho \Lambda_{\mathrm{CUT}}\right)
\end{aligned}
$$

which leads to

$$
\begin{aligned}
\Lambda_{\mathrm{SSI}}^{4} \simeq & \frac{\pi}{96} C_{\text {inst }} \Lambda_{\mathrm{diag}}^{3} \Lambda_{\mathrm{CUT}} e^{-2 \pi / \alpha_{\mathrm{CUT}}^{\prime}} \\
& \frac{3 b \alpha_{\mathrm{CUT}}^{\prime}+(b-1) \pi}{\alpha_{\mathrm{CUT}}^{\prime 6}(b-1)^{2}} \prod_{i} y_{u_{i}}^{\prime} y_{d_{i}}^{\prime} .
\end{aligned}
$$

\footnotetext{
$\overline{14}$ In Sect. 3.1.5, we will consider $y_{i}^{\prime}$ couplings of $\mathcal{O}(1)$ which corresponds to the regime of large small-size instanton effects, translating to a very heavy $\chi$ fermion which decouples from the spectrum well above the confinement regime.
}

For the benchmark $\alpha_{\text {CUT }}^{\prime}=0.3$, and substituting $N_{f}=7$, $b=16 / 3, y_{u_{i}}^{\prime}, y_{d_{i}}^{\prime}=0.2$, this gives

$\Lambda_{\mathrm{SSI}}^{4} \simeq 2.9 \times 10^{-14} \Lambda_{\mathrm{diag}}^{3} \Lambda_{\mathrm{CUT}}$

The complete computation including fermions can be compared with the one in in Eq. (37): a strong suppression by a factor of order $\left(\Lambda_{\text {diag }} / \Lambda_{\mathrm{CUT}}\right)^{3}$ is now present.

There is an additional contribution to the SSI scale independent of the the $y_{i}^{\prime}$ couplings, but suppressed by the $\chi$ chiral condensate, times the product of $\kappa_{i}$ Yukawa coupling of the prime-fermion sector in Eq. (7) and the product of SM Yukawa couplings. This contribution is illustrated by the instanton "double flower" in the right side of Fig. 2, and given by

$$
\begin{aligned}
\delta \Lambda_{\mathrm{SSI}}^{4}= & -\int \frac{d \rho}{\rho^{5}} D\left[\alpha^{\prime}(1 / \rho)\right]\left(\frac{2}{3} \pi^{2} \rho^{3}\langle\bar{\chi} \chi\rangle\right) \\
& \frac{1}{(4 \pi)^{18}} \prod_{i} Y_{u_{i}}^{S M} Y_{d_{i}}^{S M}\left(\kappa_{q}^{i}\right)^{2} \kappa_{u}^{i} \kappa_{d}^{i},
\end{aligned}
$$

where the power of the $4 \pi$ factor results from the $6 \mathrm{SM}$ Yukawa couplings and the $12 \kappa_{i}^{\prime}$ couplings in the product. For $\rho \leq 1 / \Lambda_{\mathrm{CUT}}$, this contribution is well approximated by

$$
\begin{aligned}
\delta \Lambda_{\mathrm{SSI}}^{4}= & \frac{2}{3} \frac{\pi^{2}}{(4 \pi)^{18}} C_{\text {inst }} \Lambda_{\mathrm{CUT}}^{b} e^{-\frac{2 \pi}{\alpha_{\mathrm{CUT}}^{\prime}}} \\
& \prod_{i} Y_{u_{i}}^{S M} Y_{d_{i}}^{S M} \kappa_{q}^{i^{2}} \kappa_{u}^{i} \kappa_{d}^{i} \int_{0}^{1 / \Lambda_{\mathrm{CUT}}} d \rho \rho^{b-2} \\
& \left(-b \ln \left(\rho \Lambda_{\mathrm{CUT}}\right)+\frac{2 \pi}{\alpha^{\prime}\left(\Lambda_{\mathrm{CUT}}\right)}\right)^{6} .
\end{aligned}
$$

In summary, putting together the two instanton contributions discussed, the SSI scale is given by

$$
\begin{aligned}
\Lambda_{\mathrm{SSI}}^{4}= & -\int \frac{d \rho}{\rho^{5}} D\left[\alpha^{\prime}(1 / \rho)\right]\left(\frac{2}{3} \pi^{2} \rho^{3}\langle\bar{\chi} \chi\rangle\right) \\
& \left\{\frac{1}{(4 \pi)^{6}} \prod_{i} y_{u}^{\prime i} y_{d}^{\prime i}+\frac{1}{(4 \pi)^{18}}\right. \\
& \left.\prod_{i} Y_{u_{i}}^{S M} Y_{d_{i}}^{S M}\left(\kappa_{q}^{i}\right)^{2} \kappa_{u}^{i} \kappa_{d}^{i}\right\} .
\end{aligned}
$$

Even with $\mathcal{O}(1) \kappa_{i}$ Yukawa couplings, the second term is strongly suppressed by the SM Yukawa couplings. Overall, the size of the new scale $\Lambda_{\text {SSI }}$ is quite sensitive to the value of the $S U\left(3^{\prime}\right)$ coupling constant at the CUT-breaking scale. Fig. 3 illustrates the $\eta_{\chi}^{\prime}$ axion mass induced by the small size instantons. For the benchmark examples studied, $\Lambda_{\mathrm{SSI}}$ significantly affects the properties of the pseudoscalars. It provides an additional contribution to the effective potential in Eq. (26) of the form

$$
\delta V_{e f f}=-\Lambda_{\mathrm{SSI}}^{4} \cos \left(2 \frac{\eta_{\chi}^{\prime}}{f_{\mathrm{d}}}\right)
$$




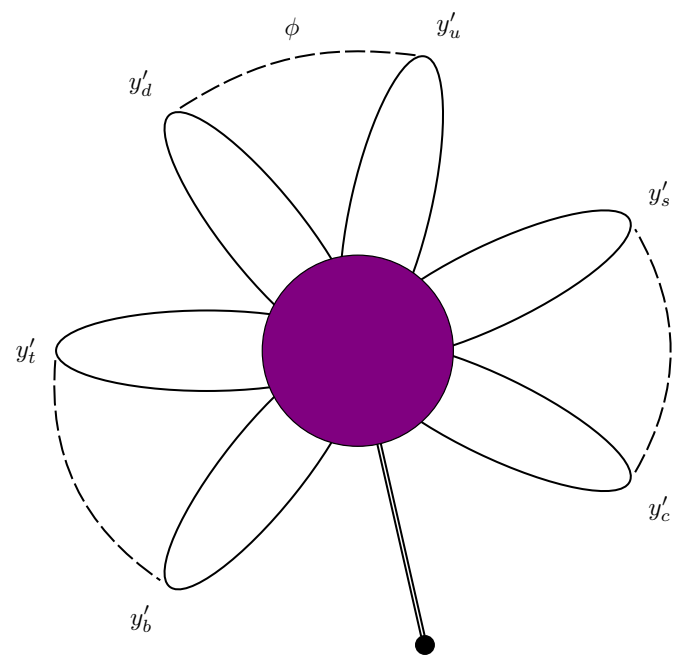

$\langle\bar{\chi} \chi\rangle$

Fig. 2 Instanton contributions in Model I. The long dashed lines connecting the $y_{i}^{\prime} \bar{q}_{L}^{\prime} \Phi u_{L}^{\prime c}$ interactions correspond to $\phi$ propagators, while the short dashed lines depict propagators of the $\Delta$ scalar. For $\mathcal{O}(1)$

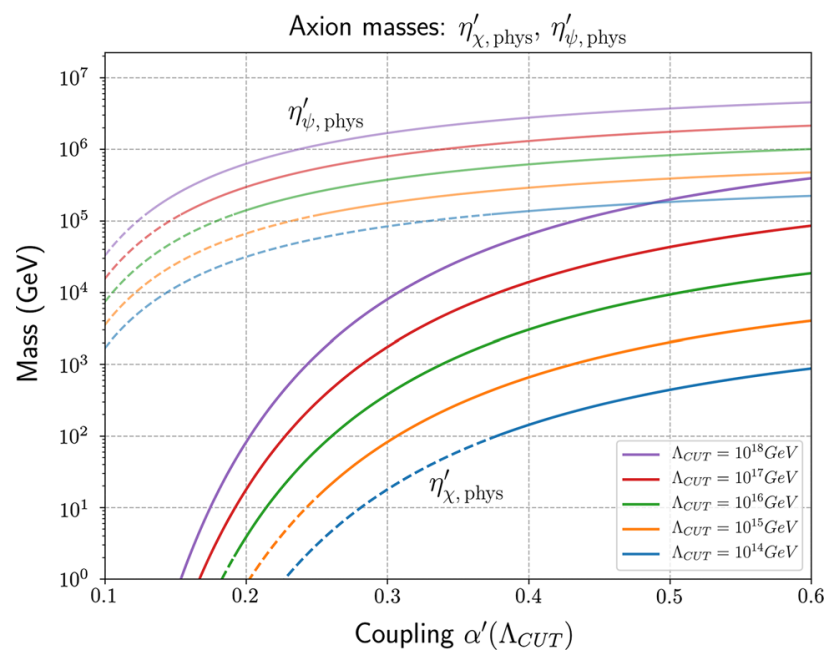

Fig. 3 Model I with small $y_{i}^{\prime}$ Yukawa couplings. The $\eta_{\chi}^{\prime}$ axion mass induced by the $S U\left(3^{\prime}\right)$ small size instantons at the scale $\Lambda_{\text {CUT }}$ is shown together with the smaller mass for the dynamical $\eta_{\psi}^{\prime}$ axion sourced by $\Lambda_{\text {diag }}$ instantons. The $\alpha_{\text {CUT }}^{\prime}$ values corresponding to solid lines are allowed, while those for dashed lines correspond to excluded values, for which $S U(3)_{\text {diag }}$ would confine below $2.9 \mathrm{TeV}$, ruled out by searches for scalar octets at LHC [45] (see Sect. 4.1)

A mass is thus generated for the $\eta_{\chi}^{\prime}$ axion, given by

$m_{\eta_{x}^{\prime}}^{2} \sim 1.8 \times 10^{-11} \Lambda_{\text {diag }} \Lambda_{\mathrm{CUT}}$,

where the replacement $\Lambda_{\text {diag }} \simeq 4 \pi f_{\mathrm{d}}$ has been used. The lighter axion may thus be as light as $\mathcal{O}(\mathrm{GeV})$. The other dynamical axion of the theory has been shown to acquire a mass of order $\Lambda_{\text {diag }}$, see Eq. (26). Both dynamical axions

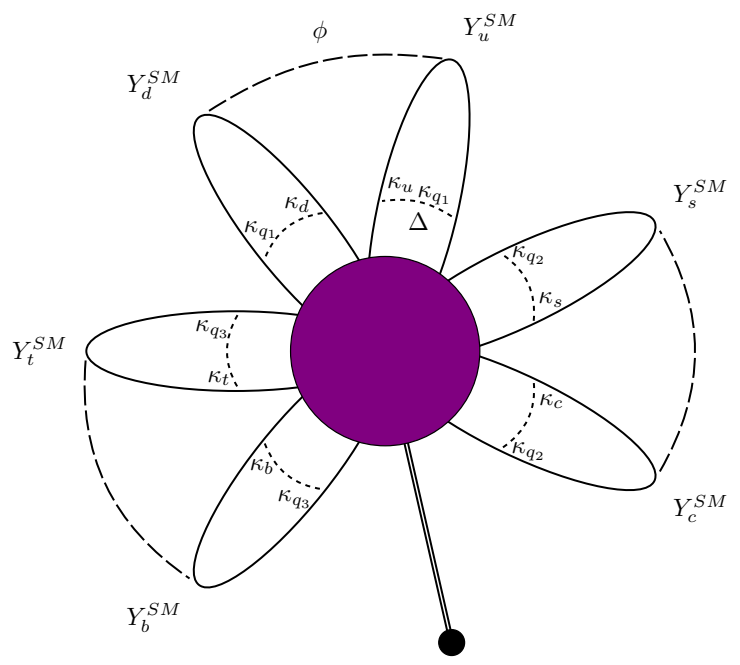

$\langle\bar{\chi} \chi\rangle$

Yukawa couplings in the prime sector, the diagram to the left represents the leading order contribution, whereas the one to the right is subdominant since it is further suppressed by the SM Yukawa couplings

have thus acquired masses far above the typical invisible axion regime, as a direct and unavoidable consequence of the instanton potentials inherent to the theory.

\section{How light can the axion that couples to SSI become?}

The $y_{i}^{\prime}$ values are very relevant for the size of SSI scale. Nevertheless, the mass spectrum and thus the running of coupling constants is basically unaffected by them. Should the $y_{i}^{\prime}$ couplings be negligible, $\Lambda_{\mathrm{SSI}}$ would be determined by the second term in Eq. (46). For vanishing $y_{i}^{\prime}$ values and generic $\kappa_{i}$ couplings of $\mathcal{O}(1)$, the product of $Y_{i}^{S M} / 4 \pi$ factors in the second term in Eq. (46) would suppress the $\eta_{\chi}^{\prime}$ axion mass to values of order keV. Such low masses are excluded up to the $\mathrm{GeV}$ range [41] for axion scales not far from a $\mathrm{TeV}$, as is the case here. The allowed range is illustrated in Fig. 3. ${ }^{15}$

\section{Solution to the strong CP problem}

It is pertinent to briefly re-check the status of the strong $\mathrm{CP}$ problem after taking into account the impact of the SSI of the spontaneously broken symmetry discussed above. Any new mass term for the axions breaks the PQ symmetry and therefore perturbs the axion potential; it is then important to verify that the vevs of the axions remain in the $\mathrm{CP}$-conserving

\footnotetext{
${ }^{15}$ For higher axion masses there are also collider constraints [41]: dijet searches at the LHC provide bounds on axions with $m_{a}>1 \mathrm{TeV}$ [42]. These searches can be extended to axion masses slightly below a $\mathrm{TeV}$ by searching for dijet resonances accompanied by hard initial state radiation [43]. These bounds are weak, though, and only apply in a small window of axion masses.
} 
minimum, solving the strong CP problem. Indeed, this is the case with our color-unified proposal as, according to Eq. (13), the potential including $\theta_{i}$ dependencies explicitly reads at second order, for the case discussed above in which both dynamical axions are present at the $S U(3)_{\text {diag }}$ confinement scale,

$$
\begin{aligned}
V_{e f f}= & \frac{\Lambda_{\mathrm{SSI}}^{4}}{2}\left(-2 \frac{\eta_{\chi}^{\prime}}{f_{\mathrm{d}}}+\bar{\theta}^{\prime}\right)^{2} \\
& +\frac{\Lambda_{\mathrm{diag}}^{4}}{2}\left(-2 \frac{\eta_{\chi}^{\prime}}{f_{\mathrm{d}}}-\sqrt{6} \frac{\eta_{\psi}^{\prime}}{f_{\mathrm{d}}}+\bar{\theta}^{\prime}+\bar{\theta}_{6}\right)^{2} \\
& +\frac{\Lambda_{\mathrm{QCD}}^{4}}{2}\left(-\sqrt{6} \frac{\eta_{\psi}^{\prime}}{f_{\mathrm{d}}}+\bar{\theta}_{6}\right)^{2} .
\end{aligned}
$$

For this potential, the minimum is $\mathrm{CP}$-conserving:

$$
\left\langle\overline{\theta^{\prime}}-2 \frac{\eta_{\chi}^{\prime}}{f_{\mathrm{d}}}\right\rangle=0, \quad\left\langle\bar{\theta}_{6}-\sqrt{6} \frac{\eta_{\psi}^{\prime}}{f_{\mathrm{d}}}\right\rangle=0,
$$

since all $\theta_{i}$ dependences cancel. A word of caution is pertinent as the exact dependence of the potential on the phases of the different couplings in the Lagrangian which participate in fermion mass generation $\left(\kappa_{i}, Y_{i}^{S M}, y_{i}^{\prime}, \ldots\right)$ remains to be computed. Nevertheless, the two massless fermions $\Psi$ and $\chi$ guarantee that at energies above CUT the two parameters $\bar{\theta}_{6}$ and $\bar{\theta}^{\prime}$ are unphysical. Below CUT, the spectrum of the theory is exclusively the SM one plus massless fermions, and the EW SM contributions are known to be negligible [44], even if a mismatch remained in spite of the low-energy presence of the massless quarks. An explicit computation of the threshold effects is elaborate and it is left for future work. Note that without the presence of the second PQ mechanism, that is, without the presence of the $\eta_{\chi}^{\prime}$ field and its vev, all $\theta_{i}$ in Eq. (13) would not have been reabsorbed, while Eq. (50) demonstrates that its inclusion does ensure a $\mathrm{CP}$-conserving minimum.

It is very positive that in this model there is no contribution to the EW hierarchy problem coming from axion physics. No potential connects the EW and axion scales: the PQ scale $f_{a}$ 16 is set by $\Lambda_{\text {diag }}$ and not $\Lambda_{\mathrm{CUT}}$, and all axions are dynamically generated. This is a feature that our Model I shares with the original axicolor model, and in general with models of composite dynamical axion(s). There remains instead the customary fine-tuning in spontaneously broken unified theories, as $\Lambda_{\mathrm{CUT}}$ and the EW scale are connected via the scalar potential, but the latter does not communicate to our PQ mechanism.

\footnotetext{
$\overline{16}$ The PQ scale (usually denoted by $f_{P Q}$ ) and $f_{a}$ differ by a modeldependent factor stemming from the relative strength of the axion coupling to gluons. Here we disregard the distinction between $f_{a}$ and $f_{P Q}$.
}

The demonstrated possibility of lowering the PQ scale towards the electroweak one raises the question of the compatibility of the setup presented here with attempts to solve the EW hierarchy problem, e.g. via compositeness or supersymmetry. This is an interesting question which deserves future work. One could probably build supersymmetric or techincolor version of the models presented here. Nevertheless, the need to strongly separate the $\Lambda_{\text {CUT }}$ scale from the EW scale is a non-trivial source of instability in the scalar potential.

Computation of the pseudoscalar mass matrix: $\eta_{\chi}^{\prime}, \eta_{\psi}^{\prime}, \eta_{\mathrm{QCD}}^{\prime}$ and light spectrum

After the replacement of the pGBs with anomalous couplings by their physical excitations, $\eta_{\chi}^{\prime} \longrightarrow\left\langle\eta_{\chi}^{\prime}\right\rangle+\eta_{\chi}^{\prime}$, $\eta_{\psi}^{\prime} \longrightarrow\left\langle\eta_{\psi}^{\prime}\right\rangle+\eta_{\psi}^{\prime}$, the effective low-energy potential for the axions and the SM $\eta_{\mathrm{QCD}}^{\prime}$ field is given at second order by (disregarding the effects of SM quark masses)

$$
\begin{aligned}
V_{e f f}= & \frac{\Lambda_{\mathrm{SSI}}^{4}}{2}\left(2 \frac{\eta_{\chi}^{\prime}}{f_{\mathrm{d}}}\right)^{2}+\frac{\Lambda_{\mathrm{diag}}^{4}}{2}\left(2 \frac{\eta_{\chi}^{\prime}}{f_{\mathrm{d}}}+\sqrt{6} \frac{\eta_{\psi}^{\prime}}{f_{\mathrm{d}}}\right)^{2} \\
& +\frac{\Lambda_{\mathrm{QCD}}^{4}}{2}\left(2 \frac{\eta_{\mathrm{QCD}}^{\prime}}{f_{\pi}}+\sqrt{6} \frac{\eta_{\psi}^{\prime}}{f_{\mathrm{d}}}\right)^{2}
\end{aligned}
$$

Expanding to second order in the fields yields the following mass matrix:

$$
M_{\eta_{\chi}^{\prime}, \eta_{\psi}^{\prime}, \eta_{\mathrm{QCD}}^{\prime}}^{2}=\left(\begin{array}{ccc}
4 \frac{\left(\Lambda_{\mathrm{SSI}}^{4}+\Lambda_{d}^{4}\right)}{f_{\mathrm{d}}^{2}} & 2 \sqrt{6} \frac{\Lambda_{d}^{4}}{f_{d}^{2}} & 0 \\
2 \sqrt{6} \frac{\Lambda_{d}^{4}}{f_{d}^{2}} & 6 \frac{\left(\Lambda_{d}^{4}+\Lambda_{\mathrm{QCD}}^{4}\right)}{f_{\mathrm{d}}^{2}} & 2 \sqrt{6} \frac{\Lambda_{\mathrm{QCD}}^{4}}{f_{\pi} f_{\mathrm{d}}} \\
0 & 2 \sqrt{6} \frac{\Lambda_{\mathrm{QCD}}^{4}}{f_{\pi} f_{\mathrm{d}}} & 4 \frac{\Lambda_{\mathrm{QCD}}^{4}}{f_{\pi}^{2}}
\end{array}\right) .
$$

Assuming the hierarchy of scales $\frac{\Lambda_{d}^{4}}{f_{\mathrm{d}}^{2}} \gg \frac{\Lambda_{\mathrm{SSI}}^{4}}{f_{\mathrm{d}}^{2}} \gg \frac{\Lambda_{\mathrm{QCD}}^{4}}{f_{\pi}^{2}}$ (valid for certain regions of parameter space, see Fig. 3), the three mass eigenvalues ${ }^{17}$ are

$m_{\eta_{\psi, p h y s}^{\prime}}^{2} \simeq 6 \frac{\Lambda_{d}^{4}}{f_{\mathrm{d}}^{2}}, \quad m_{\eta_{\chi, p h y s}^{\prime}}^{2} \simeq 4 \frac{\Lambda_{\mathrm{SSI}}^{4}}{f_{\mathrm{d}}^{2}}$

$m_{\eta_{Q C D \text { phys }}^{\prime}}^{2} \simeq 4 \frac{\Lambda_{\mathrm{QCD}}^{4}}{f_{\pi}^{2}}$.

As advertised, the usual QCD $\eta_{Q C D}^{\prime}$ phys remains as a light eigenstate, while one of the two composite axions acquires a mass in the range $\mathrm{GeV}$ to tens of $\mathrm{TeV}$. The other composite

\footnotetext{
17 The physical states correspond to the following combinations: $\eta_{\psi, p h y s}^{\prime} \simeq 1 / f_{\mathrm{d}}\left(2 \eta_{\chi}^{\prime}+\sqrt{6} \eta_{\psi}^{\prime}\right)$ and $\eta_{\chi, \text { phys }}^{\prime} \simeq 1 / f_{\mathrm{d}}\left(\sqrt{6} \eta_{\chi}^{\prime}-2 \eta_{\psi}^{\prime}\right)$.
} 
axion will have a mass orders of magnitude larger and will be out of collider reach.

\section{Low energy spectrum and observable effects}

In addition to the dynamical axion with mass $\mathcal{O}(\mathrm{GeV})$ or larger, under a few $\mathrm{TeV}$ the spectrum of the theory contains:

- The SM pseudoscalar meson $\eta_{Q C D}^{\prime}$ phys , plus the rest of the SM hadronic spectrum.

- The exotic QCD-colored "pions"- color octets and color triplets - whose masses are given in Eq. (17) as $m^{2} \sim \alpha_{c} \Lambda_{\text {diag }}^{2}$. With masses naturally lighter than the $\mathrm{TeV}$ scale, these QCD-colored pions can be easily produced at the LHC.

- The two sterile fermions stemming from the 20- representation $\Psi$. They are basically invisible as their interactions with the visible world are suppressed by $\Lambda_{\mathrm{CUT}}$, which is much larger than $\Lambda_{\text {diag }}$ without any tuning.

- Possibly, a GB associated with generalized baryon number. This GB is harmless as its interactions are suppressed by $\Lambda_{\text {CUT }}$. It can also easily be made arbitrarily heavy by gauging that global symmetry.

The very interesting phenomenological bounds and detection prospects for the exotic QCD-colored mesons will be quite similar to those applying to the next model. The ensemble will then be briefly developed in Sect. 4 further below. The same applies to the cosmological consequences of the two color-unified UV completions developed in this paper.

\subsubsection{Small-size instantons with $\mathcal{O}(1)$ Yukawa couplings}

If the Yukawa couplings in the prime sector $y_{u_{i}}^{\prime}, y_{d_{i}}^{\prime}$ are $\mathcal{O}(1)$, the instanton-induced mass of $\chi$ is larger than $\Lambda_{\text {diag. }}$. The mass of the bound state $\eta_{\chi}$, phys will be then dominated by the $\chi$ mass instead of the confinement scale. It is thus relevant to consider the instanton effects on the constituent quarks (i.e. $\chi$ ), as opposed to the discussion in the previous section in which the instantons contributed directly to the mass of the bound state $\eta_{\chi}^{\prime}$. As a consequence, the relevant diagram (Fig. 4) generates an effective mass $m_{\chi}$ for the $\chi$ quark induced by SSI instantons:

$\mathcal{L}_{e f f}=-m_{\chi} \bar{\chi} \chi$,

which can be computed using the dilute instanton gas approximation:

$$
\begin{aligned}
m_{\chi}= & \int \frac{d \rho}{\rho^{5}} D\left[\alpha^{\prime}(1 / \rho)\right]\left(\frac{2}{3} \pi^{2} \rho^{3}\right) \frac{1}{(4 \pi)^{6}} \\
& \prod_{i} y_{u}^{\prime i} y_{d}^{\prime i} \simeq 4.1 \times 10^{-10} \Lambda_{\mathrm{CUT}} .
\end{aligned}
$$

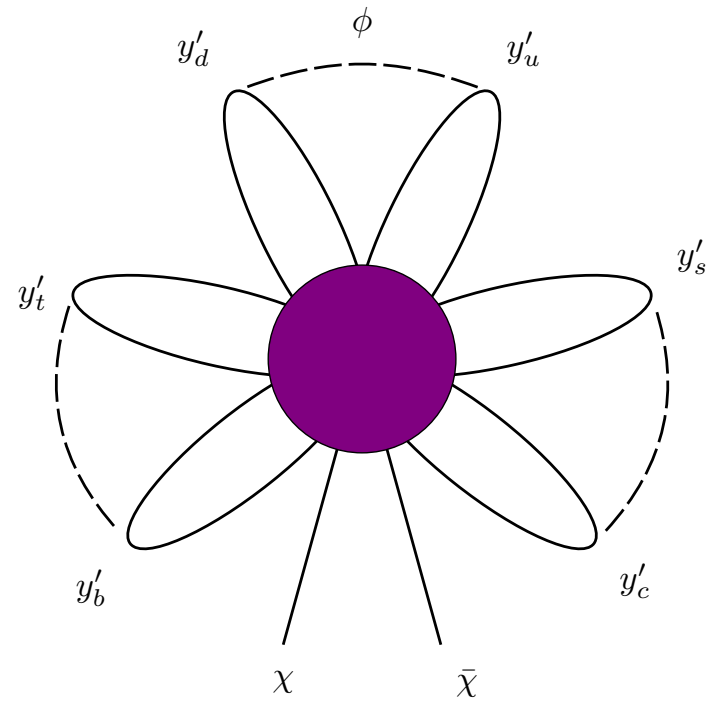

Fig. 4 Instanton contribution for the second scenario in Model I. It generates an effective mass for the $\chi$

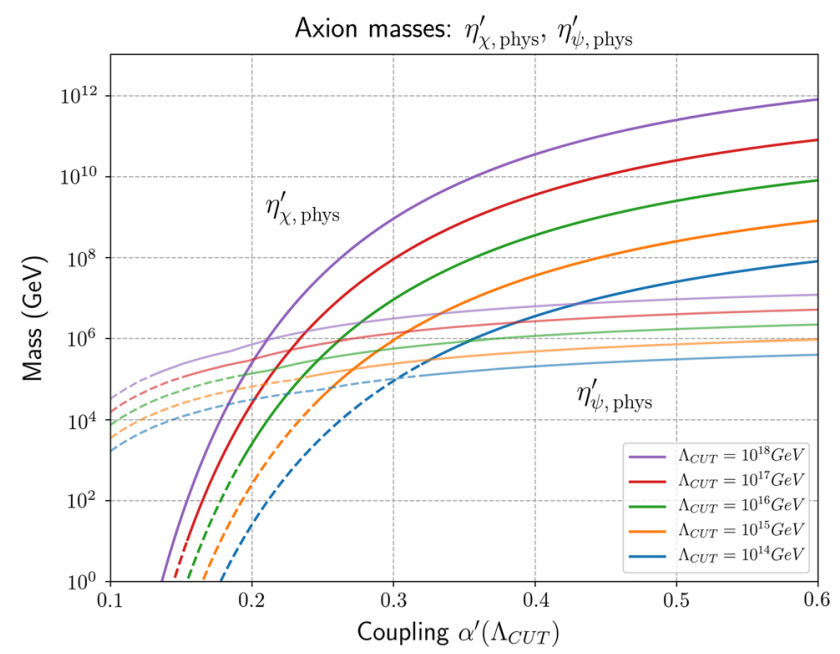

Fig. 5 Axion masses for Model I, with Yukawa couplings $y_{i}^{\prime} \sim \mathcal{O}(1)$. The slight kinks in the $\eta_{\psi}^{\prime}$ curves mark the $\chi$ threshold. The $\eta_{\chi}^{\prime}$ axion mass, dominated by the instanton effects on the constituent quarks, is shown together with the mass for the dynamical $\eta_{\psi}^{\prime}$ axion sourced by $\Lambda_{\text {diag }}$ instantons. The $\alpha_{\text {CUT }}^{\prime}$ values corresponding to solid lines are allowed, while those for dashed lines correspond to excluded values, for which $S U$ (3) diag would confine below $2.9 \mathrm{TeV}$, ruled out by searches for scalar octets at LHC [45] (see Sect. 4.1)

For the benchmark values used for $\Lambda_{\text {CUT }}$ this indicates a mass of tens of $\mathrm{TeV}$ or above. As expected, the mass of the $\chi$ thus lies well above the confinement scale $\Lambda_{d}$, and therefore the mass of the $\eta_{\chi}^{\prime}$ will be dominated by the mass of its constituent fermion: $m_{\eta_{\chi}^{\prime}} \sim 2 m_{\chi}$ The two axion masses are represented in the Fig. 5, where the mass of the $\eta_{\psi}^{\prime}$ is taken from Eq. (53) and that of $\eta_{\chi}^{\prime}$ from Eq. (55). Both composite axions have masses typically above the $\mathrm{TeV}$ regime and are out of present collider reach. 


\section{Solution to the strong CP problem}

Upon $S U(3)_{\text {diag }}$ confinement, only one massless fermion remains in the spectrum, $\psi$. Therefore, the potential contains only one pseudoscalar meson that inherits a shift symmetry: $\eta_{\psi}^{\prime}$. This does not invalidate the solution to the strong CP problem, since the other phase $\bar{\theta}^{\prime}$ in Eq. (49) is reabsorbed when the $\chi$ is integrated out of the spectrum. This is a consequence of the mass of the $\chi$ being generated by instanton effects: the phase of this mass term will be exactly that of the topological term, and it will be completely removed after integrating out the fermion.

After taking all instanton effects into account, the low energy potential for the dynamical axion now reads:

$$
\begin{aligned}
V_{e f f}= & \frac{\Lambda_{\mathrm{diag}}^{4}}{2}\left(-\sqrt{6} \frac{\eta_{\psi}^{\prime}}{f_{\mathrm{d}}}+\bar{\theta}_{6}\right)^{2} \\
& +\frac{\Lambda_{\mathrm{QCD}}^{4}}{2}\left(-\sqrt{6} \frac{\eta_{\psi}^{\prime}}{f_{\mathrm{d}}}+\bar{\theta}_{6}\right)^{2},
\end{aligned}
$$

and the minimum is $\mathrm{CP}$-conserving:

$$
\left\langle\bar{\theta}_{6}-\sqrt{6} \frac{\eta_{\psi}^{\prime}}{f_{\mathrm{d}}}\right\rangle=0,
$$

\section{Low energy spectrum and observable effects}

For most of the parameter space, the two dynamical axions $\eta_{\psi, \text { phys }}^{\prime}$ and $\eta_{\chi, \text { phys }}^{\prime}$ are typically heavier than the $\mathrm{TeV}$ scale and thus very difficult to observe at LHC. Otherwise, under a few $\mathrm{TeV}$ the spectrum is the same as that itemized in Sect. 3.1.4, except that there are no color triplet "axi-pions" because of the $\chi$ absence at the relevant energies, see Eq. (29). Therefore only the color octet "axi-pion" can be searched for at the LHC.

\subsection{Model II: Addition of a second $\Delta$ scalar.}

This solution to the $\theta^{\prime}$ problem is an alternative to extending the spectrum by a massless fermion, discussed in the previous subsection. In this second model no extra fermion is added to the $S U(6) \times S U\left(3^{\prime}\right)$ Lagrangian, while a second $\Delta$ field will be considered instead. The spectrum is that in Table 3 albeit with the scalar line duplicated, $\Delta \rightarrow\left\{\Delta_{1}, \Delta_{2}\right\}$. This simple extension allows the implementation of a PQ symmetry which reabsorbs the $\theta^{\prime}$ contribution to the strong $\mathrm{CP}$ problem. The corresponding PQ symmetry is automatic if the terms in Eq. (11) are omitted and Eq. (7) is replaced by

$\mathcal{L} \ni \kappa_{q} q_{L}^{\prime} \Delta_{1}^{*} Q_{L}+\kappa_{u} u_{L}^{\prime c} \Delta_{2} U_{L}^{c}+\kappa_{d} d_{L}^{\prime c} \Delta_{2} D_{L}^{c}+$ h.c. .
This Lagrangian is invariant under two independent abelian global symmetries; one of them is anomalous with respect to $S U\left(3^{\prime}\right)$ and corresponds to the PQ charge assignment ${ }^{18}$

$P Q\left\{\Delta_{1}, \Delta_{2}, q_{L}^{\prime}, u_{L}^{\prime c}, d_{L}^{\prime c}\right\}=\{+1,-1,+1,+1,+1\}$.

The vevs of $\Delta_{1}$ and $\Delta_{2}$ generalize the CUT spontaneous breaking in Eq. (8) and at the same time break spontaneously the PQ symmetry; therefore, this PQ scale coincides with the CUT scale. This distinguishes model 2 from Model I, as in the latter the PQ scale coincided with $\Lambda_{\text {diag. }}$. A pGB - an elementary axion - is generated at this stage. The corresponding PQ conserved current is given by

$$
\begin{aligned}
j_{P Q}^{\mu}= & {\left[\overline{q_{L}^{\prime}} \gamma^{\mu} q_{L}^{\prime}+\overline{u_{L}^{\prime c}} \gamma^{\mu} u_{L}^{\prime c}+\overline{d_{L}^{\prime c}} \gamma^{\mu} d_{L}^{\prime c}\right.} \\
& \left.+i\left(\Delta_{1} D^{\mu} \Delta_{1}^{*}-\Delta_{2} D^{\mu} \Delta_{2}^{*}-\text { h.c. }\right)\right] .
\end{aligned}
$$

The $\Delta_{i}$ fields are parameterized as

$$
\begin{aligned}
& \Delta_{1} \equiv \frac{1}{\sqrt{2}}\left(\rho_{1}+v_{\Delta_{1}}\right) e^{i a_{1} / v_{\Delta_{1}}}, \\
& \Delta_{2} \equiv \frac{1}{\sqrt{2}}\left(\rho_{2}+v_{\Delta_{2}}\right) e^{i a_{2} / v_{\Delta_{2}}},
\end{aligned}
$$

where $v_{\Delta_{1}}$ and $v_{\Delta_{2}}$ denote respectively the $\Delta_{1}$ and $\Delta_{2}$ vevs, both of which we take to be real for simplicity. Decoupling the heavy radial modes, the PQ current reads

$j_{P Q}^{\mu} \supset v_{\Delta_{1}} \partial^{\mu} a_{1}-v_{\Delta_{2}} \partial^{\mu} a_{2} \equiv f_{a} \partial^{\mu} a$,

where the elementary axion field $a(x)$ corresponds to the GB combination

$a(x)=\frac{1}{f_{a}}\left(v_{\Delta_{1}} a_{1}(x)-v_{\Delta_{2}} a_{2}(x)\right)$,

with

$f_{a}=\Lambda_{\mathrm{CUT}}=\sqrt{v_{\Delta_{1}}^{2}+v_{\Delta_{2}}^{2}}$.

This classically exact PQ symmetry is broken at the quantum level by the $S U\left(3^{\prime}\right)$ anomaly, which at lower energies translates into an anomalous current for the $S U(3)_{\text {diag gauge }}$ theory.

$\partial_{\mu} j_{P Q}^{\mu}=\frac{\alpha^{\prime}}{8 \pi} N^{\prime} G^{\prime} \tilde{G}^{\prime} \longrightarrow \frac{\alpha_{\text {diag }}}{8 \pi} N_{\text {diag }} G_{\text {diag }} \tilde{G}_{\text {diag }}$,

where $N^{\prime}$ and $N_{\text {diag }}$ are the group factors,

$N^{\prime}=N_{\text {diag }}=\sum_{L H-R H} \operatorname{Tr}\left[T_{P Q}^{a}\left\{t^{b}, t^{c}\right\}\right]=12$,

and where $T_{P Q}^{a}$ corresponds to the PQ generator and $t^{b}=\frac{\lambda^{b}}{2}$ to the Gell-Mann matrices for the $S U(3)$ generators. The

\footnotetext{
18 The Lagrangian possesses another $U(1)$ symmetry, namely the generalized baryon number symmetry defined in Eq. (12), which is however non-anomalous under $S U\left(3^{\prime}\right)$. See footnotes 8 and 9 on the harmless consequences of the non-anomalous global symmetry.
} 
Table 6 The massless quark sector charged under $S U(3)_{\text {diag }}$ below $\Lambda_{\text {CUT }}$ in the model with an extra scalar

anomalous term modifies the classical equations of motion of the axion,

$$
\begin{aligned}
f_{a} & \square a+\partial_{\mu}\left(\overline{q_{L}^{\prime}} \gamma^{\mu} q_{L}^{\prime}\right)+\partial_{\mu}\left(\overline{u_{L}^{\prime c}} \gamma^{\mu} u_{L}^{\prime c}\right)+\partial_{\mu}\left(\overline{d_{L}^{\prime c}} \gamma^{\mu} d_{L}^{\prime c}\right) \\
= & \frac{\alpha_{\text {diag }}}{8 \pi} 12 G_{\text {diag }} \tilde{G}_{\text {diag }},
\end{aligned}
$$

and gives rise to an effective Lagrangian,

$$
\begin{aligned}
\mathcal{L} \supset & \frac{1}{2} \partial_{\mu} a \partial^{\mu} a \\
& +\frac{1}{f_{a}} \partial_{\mu} a\left(\overline{q_{L}^{\prime}} \gamma^{\mu} q_{L}^{\prime}+\overline{u_{L}^{\prime c}} \gamma^{\mu} u_{L}^{\prime c}+\overline{d_{L}^{\prime c}} \gamma^{\mu} d_{L}^{c}\right) \\
& +\frac{\alpha_{\text {diag }}}{8 \pi} \frac{12}{f_{a}} a G_{\text {diag }} \tilde{G}_{\text {diag }} .
\end{aligned}
$$

The impact of the SSI of the spontaneously broken theory will again add further contributions, inducing a putatively high mass for the elementary axion as discussed further below.

\subsubsection{Running of the coupling constants}

The matter content allows $S U(3)_{\text {diag }}$ to confine at higher scales than the QCD group $S U(3)_{c}$ as in Model I. The separation of both scales is made even sharper in the Model II because $\alpha_{\text {diag }}$ runs faster. In Model II, only one massless fermion charged under $S U(3)_{\text {diag }}$ is ever present under the CUT scale (compare Tables 5 and 6). We have estimated both the one and two-loop running, as illustrated in Fig. 6 for $\Lambda_{\text {diag }}=4 \mathrm{TeV}$.

\subsubsection{Confinement of $S U(3)_{\mathrm{diag}}$ and pseudoscalar anomalous couplings to the confining interactions.}

At the scale $\Lambda_{\text {diag }}$ the QCD coupling constant is small, and the $S U(3)_{\text {diag }}$ spectrum with only one massless fermion in Table 6 has an approximate classical global symmetry $U(3)_{L} \times U(3)_{R}$. Upon chiral symmetry breaking $U(3)_{L} \times$ $U(3)_{R} \longrightarrow U(3)_{V}$ by the quark condensate $\left\langle\bar{\psi}_{L} \psi_{R}\right\rangle$, nine pGBs appear,

$9=1_{c}+8_{c}$

The gauge QCD group $S U(3)_{c}$ is again a subgroup of $U(3)_{V}$ which remains unbroken. The octet of pGBs colored under QCD will acquire large masses due to gluon loops, which at one-loop is given by

$m^{2}\left(8_{c}\right) \approx \frac{9 \alpha_{\mathrm{c}}}{4 \pi} \Lambda_{\mathrm{diag}}^{2}$

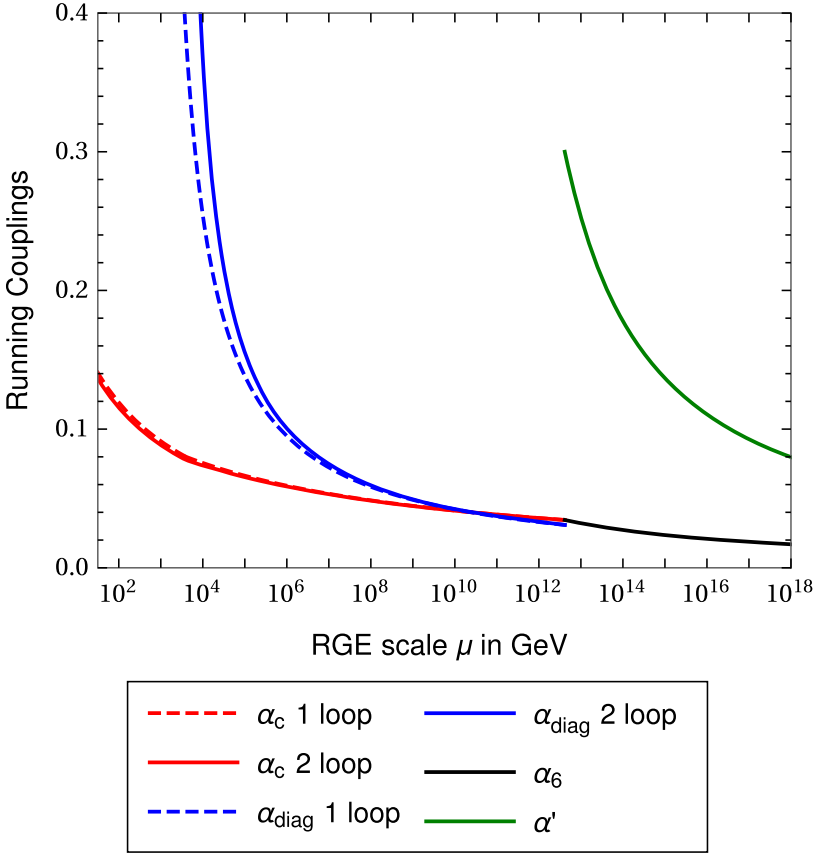

Fig. 6 Running of $\alpha_{\mathrm{QCD}}, \alpha_{\text {diagonal }}, \alpha_{6}$, and $\alpha^{\prime}$ in the model with one extra $S U(6)$ scalar; the only inputs assumed are $\alpha_{\text {CUT }}^{\prime}=0.3$ and $\Lambda_{\mathrm{CUT}}=4.1 \times 10^{12} \mathrm{GeV}$ for illustration, which results in $\Lambda_{\text {diag }}=4 \mathrm{TeV}$ (taken as a benchmark point). The solid (dashed) lines correspond to the two (one) loop results

The QCD singlet $1_{c}$, denoted $\eta_{\psi}^{\prime}$, is a dynamical axion. Note that it has the same quark composition as the $\eta_{\psi}^{\prime}$ meson in Model I. The $\eta_{\psi}^{\prime}$ couples to both the $S U(3)_{\text {diag }}$ and $S U(3)_{c}$ anomalies,

$j_{\psi_{A}}^{\mu}=\bar{\psi} \gamma^{\mu} \gamma^{5} t^{9} \psi \equiv f_{\mathrm{d}} \partial^{\mu} \eta_{\psi}^{\prime}, \quad t^{9}=\frac{1}{\sqrt{6}} \mathbb{1}_{3 \times 3}$,

resulting in a low-energy effective Lagrangian for this axion given by

$\mathcal{L}_{e f f} \subset-\frac{\sqrt{6} \eta_{\psi}^{\prime}}{f_{\mathrm{d}}}\left(\frac{\alpha_{s}}{8 \pi} G_{c} \tilde{G}_{c}+\frac{\alpha_{\mathrm{diag}}}{8 \pi} G_{\mathrm{diag}} \tilde{G}_{\mathrm{diag}}\right)$.

In summary, this solution to the strong $\mathrm{CP}$ problem is a hybrid one with two axions: a heavy dynamical axion $\eta_{\psi}^{\prime}$ with mass of order $\Lambda_{\text {diag }}$ stemming from a PQ symmetry which reabsorbs the original $\theta_{S U(6)}$ (and thus $\theta_{\mathrm{QCD}}$ ) parameter as in the previous section, and a second elementary axion $a$ resulting from solving the external $S U\left(3^{\prime}\right)$ sector à $l a \mathrm{PQWW}$ $[12,13,46]$. Up to now, only two sources of masses have been identified for the ensemble of three pseudoscalars with anomalous couplings $\left(\eta_{\mathrm{QCD}}^{\prime}, \eta_{\psi}^{\prime}\right.$ and $a$ ). We analyze next the SSI of this model which provide a large source of axion mass for the elementary axion $a$. 


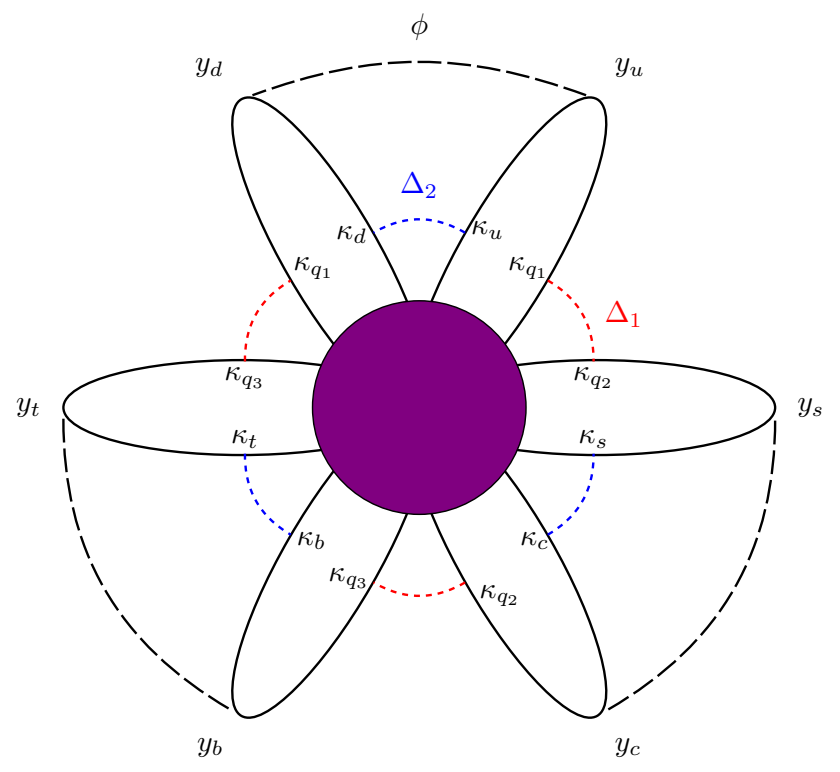

Fig. 7 Instanton contribution in Model II. The long dashed lines connecting the SM Yukawa interactions correspond to $\phi$ propagators while the short dashed lines depict $\Delta_{1}$ or $\Delta_{2}$ propagators

\subsubsection{Impact of small-size instantons on the dynamical axion mass}

\section{Small-size instantons of the spontaneously broken CUT}

The analysis of SSI for Model II under discussion is simpler than that for Model I developed in the previous subsection. $N o$ massless fermions charged under $S U\left(3^{\prime}\right)$ are present in Model II (in contrast with Model I). Furthermore, PQ symmetry forbids here the $y_{i}^{\prime}$ Yukawa couplings which gave the dominant contribution in Model I. In consequence, the terms proportional to $\kappa_{i}$ and mediated by the $\Delta_{1}$ and $\Delta_{2}$ scalars and the Higgs field will dominate $\Lambda_{\mathrm{SSI}}$. This is illustrated by the instanton "flower" in Fig. 7. It results in $\Lambda_{\text {SSI }}$ given by

$$
\begin{aligned}
\Lambda_{\mathrm{SSI}}^{4}= & -\int \frac{d \rho}{\rho^{5}} D\left[\alpha^{\prime}(1 / \rho)\right] \frac{1}{(4 \pi)^{18}} \\
& \prod_{i} Y_{u_{i}}^{S M} Y_{d_{i}}^{S M}\left(\kappa_{q}^{i}\right)^{2} \kappa_{u}^{i} \kappa_{d}^{i},
\end{aligned}
$$

which can be written as

$$
\begin{aligned}
\Lambda_{\mathrm{SSI}}^{4}= & C_{i n s t} \Lambda_{\mathrm{CUT}}^{b} e^{-\frac{2 \pi}{\alpha_{\mathrm{CUT}}^{\prime}}} \frac{1}{(4 \pi)^{18}} \\
& \prod_{i} Y_{u_{i}}^{S M} Y_{d_{i}}^{S M} \kappa_{q}^{i^{2}} \kappa_{u}^{i} \kappa_{d}^{i} \int_{0}^{1 / \Lambda_{\mathrm{CUT}}} d \rho \rho^{b-5} \\
& \left(-b \ln \left(\rho \Lambda_{\mathrm{CUT}}\right)+\frac{2 \pi}{\alpha^{\prime}\left(\Lambda_{\mathrm{CUT}}\right)}\right)^{6}
\end{aligned}
$$

with $b=5$ in this case. Here the approximation in Eq. (41) is no longer valid, and the result corresponds to that of the pure Yang-Mills case (Eq. 35) with the extra suppression factor of the Yukawa couplings,

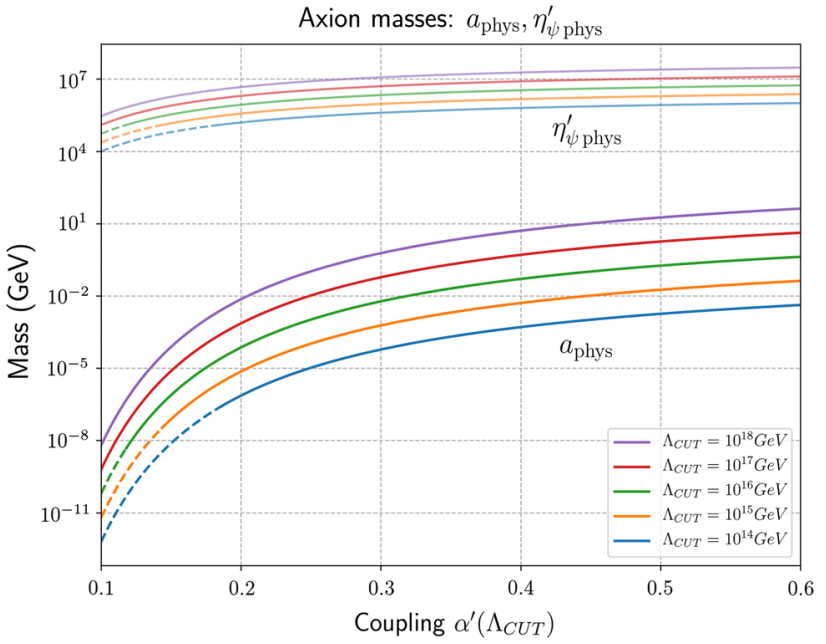

Fig. 8 Model with a second $S U(6)$ scalar. The $a$ axion mass induced by the $S U\left(3^{\prime}\right)$ small size instantons at the scale $\Lambda_{\mathrm{CUT}}$ is illustrated, together with the larger mass for the dynamical axion $\eta_{\psi}^{\prime}$ sourced by $S U(3)_{\text {diag }}$ instantons. Order one Yukawa couplings in the prime sector have been used in this figure. The $\alpha_{\text {CUT }}^{\prime}$ values allowed correspond to the solid sectors of the lines, while dashed lines correspond to excluded values, for which $S U(3)_{\text {diag }}$ would confine below $2.9 \mathrm{TeV}$, ruled out by searches for scalar octets at LHC [45] (see Sect. 4.1)

$$
\begin{aligned}
\Lambda_{\mathrm{SSI}}^{4}= & C_{\text {inst }} f\left(\alpha_{\mathrm{CUT}}^{\prime}, b\right) e^{-\frac{2 \pi}{\alpha_{\mathrm{CUT}}^{\prime}}} \Lambda_{\mathrm{CUT}}^{4} \frac{1}{(4 \pi)^{18}} \\
& \prod_{i} Y_{u_{i}}^{S M} Y_{d_{i}}^{S M} \kappa_{q}^{i^{2}} \kappa_{u}^{i} \kappa_{d}^{i},
\end{aligned}
$$

where the function $f\left(\alpha_{\mathrm{CUT}}^{\prime}, b\right)$ is defined in Eq. (36).

This result translates into a new contribution to the instanton-induced effective potential of the form

$\delta V_{e f f}=-\Lambda_{\mathrm{SSI}}^{4} \cos \left(12 \frac{a}{f_{a}}\right)$.

Taking into account that in this model the elementary axion scale coincides with the CUT scale, it follows that for $\alpha_{\text {CUT }}^{\prime}=$ 0.3 and with $\mathcal{O}(1) \kappa_{i}$ Yukawa couplings,

$m_{a}^{2} \sim 3.7 \times 10^{-37} \Lambda_{\mathrm{CUT}}^{2}$.

For the benchmark values used for $\Lambda_{\mathrm{CUT}}$, this implies an elementary axion mass in the range $10^{-5}-1 \mathrm{GeV}$, see Fig. 8 for illustration. In summary, the dynamical axion has a mass of order $\Lambda_{\text {diag }}$ and thus of a few TeV or above, while the elementary axion is light although typically heavier than the usual invisible axion.

\section{Solution to the strong CP problem}

The minimum of the axion potential can be easily shown to remain $\mathrm{CP}$-conserving after including all contributions to the axion masses. Indeed, the $\theta_{i}$ dependence of the potential can be again read off of Eq. (13), 


$$
\begin{aligned}
V_{e f f}= & \frac{\Lambda_{\mathrm{SSI}}^{\prime 4}}{2}\left(12 \frac{a}{f_{a}}+\bar{\theta}^{\prime}\right)^{2} \\
& +\frac{\Lambda_{\mathrm{d}}^{4}}{2}\left(12 \frac{a}{f_{a}}-\sqrt{6} \frac{\eta_{\psi}^{\prime}}{f_{\mathrm{d}}}+\bar{\theta}^{\prime}+\bar{\theta}_{6}\right)^{2} \\
& +\frac{\Lambda_{\mathrm{QCD}}^{4}}{2}\left(-\sqrt{6} \frac{\eta_{\psi}^{\prime}}{f_{\mathrm{d}}}+\bar{\theta}_{6}\right)^{2}
\end{aligned}
$$

for which the following bosonic vevs lead to a CP-conserving minimum,

$$
\left\langle 12 \frac{a}{f_{a}}+\bar{\theta}^{\prime}\right\rangle=0, \quad\left\langle\bar{\theta}_{6}-\sqrt{6} \frac{\eta_{\psi}^{\prime}}{f_{\mathrm{d}}}\right\rangle=0
$$

After the replacement $a \longrightarrow\langle a\rangle+a, \eta_{\psi}^{\prime} \longrightarrow\left\langle\eta_{\psi}^{\prime}\right\rangle+\eta_{\psi}^{\prime}$ and introducing as well the QCD $\eta_{\mathrm{QCD}}^{\prime}$ field, the effective low-energy potential for the physical mesons which couple to anomalous currents is given at second order by

$$
\begin{aligned}
V_{e f f}= & \frac{\Lambda_{\mathrm{SSI}}^{4}}{2}\left(12 \frac{a}{f_{a}}\right)^{2}+\frac{\Lambda_{\mathrm{d}}^{4}}{2}\left(12 \frac{a}{f_{a}}-\sqrt{6} \frac{\eta_{\psi}^{\prime}}{f_{\mathrm{d}}}\right)^{2} \\
& +\frac{\Lambda_{\mathrm{QCD}}^{4}}{2}\left(2 \frac{\eta_{\mathrm{QCD}}^{\prime}}{f_{\pi}}+\sqrt{6} \frac{\eta_{\psi}^{\prime}}{f_{\mathrm{d}}}\right)^{2} .
\end{aligned}
$$

where all the $\mathrm{CP}$ violating phases have been relaxed to zero.

\section{Computation of the pseudoscalar mass matrix: $a, \eta_{\psi}^{\prime}, \eta_{\mathrm{QCD}}^{\prime}$ and light spectrum}

Taking into account all contributions except the SM quark masses, the following mass matrix results for the singlet pseudoscalars of the theory which couple to anomalous currents:

$$
\begin{aligned}
& M_{a, \eta_{\psi}^{\prime}, \eta_{\mathrm{QCD}}^{\prime}}^{2} \\
& =\left(\begin{array}{ccc}
144 \frac{\left(\Lambda_{\mathrm{SSI}}^{4}+\Lambda_{\mathrm{diag}}^{4}\right)}{f_{a}^{2}} & -12 \sqrt{6} \frac{\Lambda_{d}^{4}}{f_{\mathrm{d}} f_{a}} & 0 \\
-12 \sqrt{6} \frac{\Lambda_{d}^{4}}{f_{\mathrm{d} f} f_{a}} & 6 \frac{\left(\Lambda_{d}^{4}+\Lambda_{\mathrm{QCD}}^{4}\right)}{f_{\mathrm{d}}^{2}} & 2 \sqrt{6} \frac{\Lambda_{\mathrm{QCD}}^{4}}{f_{\pi} f_{\mathrm{d}}} \\
0 & 2 \sqrt{6} \frac{\Lambda_{\mathrm{QCD}}^{4}}{f_{\pi} f_{\mathrm{d}}} & 4 \frac{\Lambda_{\mathrm{QCD}}^{4}}{f_{\pi}^{2}}
\end{array}\right) .
\end{aligned}
$$

Assuming the hierarchy of scales $\frac{\Lambda_{d}^{4}}{f_{\mathrm{d}}^{2}} \gg \frac{\Lambda_{\mathrm{QCD}}^{4}}{f_{\pi}^{2}} \gg \frac{\Lambda_{\mathrm{SSI}}^{4}}{f_{a}^{2}}$, the resulting mass eigenvalues are

$$
\begin{aligned}
& m_{a_{p h y s}}^{2} \simeq 144 \frac{\Lambda_{\mathrm{SSI}}^{4}}{f_{a}^{2}}, \quad m_{\eta_{\psi, p h y s}^{\prime}}^{2} \simeq 6 \frac{\Lambda_{d}^{4}}{f_{\mathrm{d}}^{2}}, \\
& m_{\eta_{Q C D p h y s}^{\prime}}^{2} \simeq 4 \frac{\Lambda_{\mathrm{QCD}}^{4}}{f_{\pi}^{2}} .
\end{aligned}
$$

In this model both the usual QCD $\eta^{\prime}$ and the axion $a_{p h y s}$ remain as light eigenstates, while the other eigenstate $\eta_{\psi \text {, phys }}^{\prime}$ will have a mass generically above the $\mathrm{TeV}$ scale.

Apart from the axion, the lowest set of exotic states is an octet of exotic "pions" whose masses are $\lesssim \mathrm{TeV}$, see Eq. (70). A similar colored octet is present in Model I discussed in Sect. 3.1, although Model I contains an additional set of color-triplet pseudoscalars in the case of small $y_{i}^{\prime}$ Yukawa couplings. In contrast, no color-triplet is ever expected here as the exotic classical flavor symmetry is $U(3)_{L} \times U(3)_{R}$, see Eq. (69), instead of the $U(4)_{L} \times U(4)_{R}$ symmetry of Model I.

This Model II with an additional scalar may be less appealing than than Model I with an extra massless fermions for two reasons: (a) its axion sector contributes directly to the EW hierarchy problem, as its elementary axion results from a scalar potential which a priori communicates with the Higgs potential; (b) it is a hybrid model with both one elementary and one dynamical axion, while Model I is more aligned with the spirit of solving fully the strong CP problem via massless fermions.

\section{Phenomenological and cosmological limits on the lightest exotic states}

A common feature of the ultraviolet complete models constructed above is that the generic spectrum under the EW scale includes, in addition to the SM spectrum, sterile fermions, in contrast with usual axion models. An axion may also be present in this range depending on the model parameters.

\subsection{Collider observable signals}

A set of observable exotic states are expected to be the exotic $S U(3)_{c}$-colored "pions" whose masses may lie under the $\mathrm{TeV}$ scale. These resulted from the chiral symmetry breaking of the confining group $S U(3)_{\text {diag. }}$. All models exhibit as a common characteristic QCD color-octet meson bound states made out of their massless fermions, shown in Table 5 and Eq. (17) for Model I and Table 6 and Eq. (70) for Model II. In addition, QCD color-triplet meson bound states may be observable for Model I with small Yukawa couplings in the primed sector.

In this color-unified axion solution, the exotic fundamental fermions have no SM $S U(2) \times U(1)$ charges. The heavy pions will be produced in colliders only via QCD interactions, e.g. gluon-gluon couplings, through which they also presumably decay before they can hadronize to make color neutral states. As they are colored, they do not mix with ordinary pions or other visible matter. 


\subsubsection{Color-octet pions from $S U(3)_{\mathrm{diag}}$ confinement}

The lightest scalar octets in Eqs. (17) and (70) are denoted by $\pi_{\mathrm{d}} \equiv 8_{c}$. Their effective coupling to QCD gluons can be written as

$\mathcal{L} \supset D_{\mu} \pi_{\mathrm{d}} D^{\mu} \pi_{\mathrm{d}}+\frac{3 \sqrt{3} \alpha_{s}}{8 \pi} \frac{\pi_{\mathrm{d}}^{a}}{f_{d}} d_{a b c} G_{\mu \nu}^{b} \tilde{G}^{c \mu \nu}$,

where $D_{\mu}$ denotes the $S U(3)_{c}$ covariant derivative and $d_{a b c}$ is the corresponding symmetric group structure constant. The second term in Eq. (83) results at one-loop from the triangle diagram with the fermions $\chi$ running in the loop. The coloroctet exotic pions can thus be either produced in pairs through the gluon-gluon- $\pi_{d}-\pi_{d}$ coupling in the kinetic term, or singly produced through their anomalous coupling to gluons. The kinetic term dominates the scalar octet production channels, while the second term allows the $\pi_{\mathrm{d}}$ decay, yielding a dijet final state.

Experimental limits on scalar octet pair production via the gluonic interactions in the kinetic term can be inferred by recasting searches of sgluons. A recent search of sgluon pair production by ATLAS using $36.7 \mathrm{fb}^{-1}$ of $\sqrt{s}=13 \mathrm{TeV}$ data [45], whose prediction was obtained from the NLO computation in Ref. [47] for $\sqrt{s}=8 \mathrm{TeV}$ (rescaled to $13 \mathrm{TeV}$ according to Ref. [48]), sets a bound on the octet scalar exotic pions given by

$m\left(\pi_{\mathrm{d}}\right) \gtrsim 770 \mathrm{GeV}$.

From Eqs. (17) and (70), this translates into

$\Lambda_{\text {diag }} \gtrsim 2.9 \mathrm{TeV}$.

For Model I and in the particular case of small Yukawa couplings in the primed sector, an alternative bound may be inferred from the limits on color-triplet scalars, which can be produced via their color interactions. In the absence of couplings which mediate their decay (as the second type of coupling in Eq. (83) is not possible for scalar triplets), they will bind with SM quarks to form stable hadrons. This search is expected to result in a sensitivity similar to the one above [49].

It is very interesting to pursue the experimental search for colored pseudoscalars and stable exotic hadrons. Their detection would be a powerful indication of the dynamical solution to the strong $\mathrm{CP}$ problem proposed here.

\subsubsection{Dynamical axion and exotic fermions}

The dynamical axion denoted above by $\eta_{\psi, \text { phys }}^{\prime}$ with instanton-induced mass of order $\Lambda_{\text {diag }}$, Eqs. (53) and (82), can a priori be either pair-produced through the kinetic coupling or singly produced through the dimension five anomalous operator. It would decay dominantly to two back-to-back jets and can be searched for in dijet resonance searches. Its production, however, may be suppressed by its high mass. For instance, for $\Lambda_{\text {diag }} \simeq 2.9 \mathrm{TeV}, m_{\eta_{\psi, p h y s}^{\prime}} \sim 90 \mathrm{TeV}$, which is beyond the reach of present collider searches. The other mesons and baryons resulting from the $S U(3)_{\text {diag }}$ confinement have masses in the $\mathrm{TeV}$ range and above; they would lead to collider signatures similar to those for the exotic pseudo-goldstone bosons.

\subsubsection{The axion coupled to SSI}

In Model I, the second axion is also dynamical and it was denoted by $\eta_{\chi, \text { phys }}^{\prime}$. Its mass can span a large range, depending on the strength of the Yukawa couplings in the prime sector:

- For $y_{i}^{\prime}$ couplings of $\mathcal{O}(1)$, this second axion becomes typically heavier than the $\mathrm{TeV}$ scale and is thus out of LHC reach.

- For small $y_{i}^{\prime}$ couplings $(\mathcal{O}(0.2)$ have been illustrated $)$, an observable axion with mass in the $\mathrm{GeV}$-tens of $\mathrm{TeV}$ regime is realistic. Given the low PQ scale $\left(f_{d}\right.$, close to a $\mathrm{TeV}$ ) lower masses are excluded.

The second axion in Model II, denoted $a$ and of elementary nature, is expected to be light although heavier than the invisible axion, with a mass in the $10 \mathrm{eV}-10 \mathrm{GeV}$ range for $\mathcal{O}(1)$ Yukawa couplings in the prime-fermion sector. By lowering the values of the latter couplings, it can even become as light as the usual invisible axion and with similar phenomenology, as its associated axion scale is the color-unified scale, $f_{a} \sim \Lambda_{\text {CUT }}$.

\subsection{Cosmological and gravitational aspects}

We briefly discuss next the cosmological aspects of the models constructed above, as well as the putative instability threat from gravitational non-perturbative effects.

\subsubsection{Stable particles and cosmological structures}

Stable particles with masses higher than about $10^{5} \mathrm{GeV}$ may lead to cosmological problems, dominating the mass density and overclosing the universe [50]. This is often a problem in previous models of composite axions because exotic stable baryons bound by the extra confining force $[11,51]$ are expected.

However, as pointed out in Refs. [51,52], if axicolor can be unified with a SM gauge group, then the unified forces could mediate the decay of axihadrons into lighter states, and the model would be cosmologically safe. The color unification of this work automatically employs this mechanism. The heavy exotic hadrons decay to the sterile fermions $\psi_{\nu}$, which are 
part of the CUT massless multiplet $\Psi$ in Eq. (4) and Table 2. It remains to be determined whether the lifetime for these CUT-induced decay channels is too large to avoid problems from stable exotic hadrons. If the decays are made to be fast enough, the resulting sterile fermions may induce in turn other cosmological problems. This analysis is left to a future work.

A similar concern pertains to the domain walls which may arise due to the spontaneous breaking of the discrete shift symmetry in the instanton potential, Eqs. (51) and (80). The walls need to disappear before they dominate the matter density of the universe, or else other mechanisms must be applied to solve the domain wall problem [53-57].

In any case, if the universe went through an inflation phase any relic previously present will be wiped out. If the reheating temperature is lower than the PQ scales, meaning lower than $\Lambda_{\text {diag }}$ here, neither the heavy stable particles nor any putative domain walls are produced again after inflation, and the problems mentioned above are avoided altogether. We defer to a future work the in-depth study of the cosmological aspects of Model I and Model II, with either high- or low-scale inflation.

\subsubsection{The question of gravitational quantum effects}

Gravitational quantum corrections have been suggested to be relevant and dangerous for axion models in which the PQ scale is not far from the Planck scale. In Model I, both PQ scales correspond to $\Lambda_{\text {diag }}$, which is much lower than the Planck scale, and no instability resulting from gravitational quantum effects is at stake. In Model II instead, while the dynamical PQ scale is analogously low, the second PQ symmetry is realized at the CUT scale and gravitational quantum effects could be relevant.

It has often been argued that all global symmetries may be violated by non-perturbative quantum gravitational effects, see for instance Refs. [54,58-61]. For instance, a black hole can eat global charges and subsequently evaporate. Similar effects may exist with virtual black holes. Another indication that gravity might not respect global symmetries comes from wormhole physics [62-65]. The natural scale of violation in this case is the wormhole scale, usually thought to be very near (within an order of magnitude or so) the Planck mass MPlanck.

For axion models with high PQ scales, such as the typical scale of invisible axion models $f_{a} \sim 10^{9}-10^{12} \mathrm{GeV}$, it has been argued that those non-perturbative quantum gravitational effects could lead to extreme fine-tunings. The authors of Ref. [54,58-60] concentrated on the simplest (and the most dangerous) hypothetical dimension five effective operator

$g_{5} \frac{|\Phi|^{4}\left(\Phi+\Phi^{*}\right)}{M_{\text {Planck }}}$, where $g_{5}$ is a dimensionless coefficient and $\Phi$ would be a field whose vev breaks the PQ invariance. This term threatens the standard invisible axion solutions to the strong $\mathrm{CP}$ problem, as it would change the shape of the effective potential. The minimum moves unacceptably away from a $\mathrm{CP}$ conserving solution unless the coefficient is strongly finetuned, for instance $g_{5}<10^{-54}$ for $f_{a} \sim 10^{12} \mathrm{GeV}$. These potentially dangerous terms can be avoided if the global PQ symmetry arises accidentally as a consequence of other gauge groups $[66,67]$. Nevertheless, the idea that gravity breaks all global symmetries is indeed an assumption - and sometimes an incorrect one - at least at the Lagrangian level. ${ }^{19}$ Furthermore, very recently the impact of non-perturbative effects has been clarified and quantified in Ref. [69]. The effects may be extremely suppressed by an exponential dependence on the gravitational instanton action, and be harmless even with high axion scales. The demonstration relied on assuming that the spontaneous breaking of the PQ symmetry is implemented through the vev of a scalar field, and thus it directly applies to our Model II. ${ }^{20}$ In summary, Model I is safe from instabilities induced by gravitational quantum corrections and plausibly this also applies to Model II.

\section{Conclusions}

Color unification with massless quarks has been proposed and developed here for the first time. As a simple implementation of the idea, the SM color group has been embedded in $S U$ (6), which is spontaneously broken to QCD and a second confining and unbroken gauge group. An exactly massless $S U$ (6) fermion multiplet solves the strong CP problem. We have fully developed two ultraviolet completions of the mechanism.

In order to implement this idea successfully, it is necessary to give satisfactorily high masses to the $S U(6)$ partners of the SM quarks to achieve a separation between the QCD scale and that of the second confining group. For this purpose, an auxiliary $S U\left(3^{\prime}\right)$ gauge group is introduced under which the aforementioned massless fermion is a singlet. $S U(6) \times S U\left(3^{\prime}\right) \longrightarrow S U(3)_{c} \times S U(3)_{\text {diag }}$ is a simple and realistic option. Both final groups remain unbroken and confine at two different scales, $\Lambda_{\mathrm{QCD}}$ and $\Lambda_{\text {diag, }}$, with $\Lambda_{\text {diag }} \sim \mathcal{O}(\# \mathrm{TeV}) \gg \Lambda_{\mathrm{QCD}}$. The scale $\Lambda_{\text {diag }}$ then gives the order of magnitude of the mass of the dynamical composite axion inherent to the color-unified mechanism. Further-

\footnotetext{
${ }^{19}$ For example, orbifold compactifications of the heterotic string have discrete symmetries that prevent the presence of some higher dimension operators, and this can strongly and safely suppress the dangerous effects under discussion [68].

${ }^{20}$ Although no explicit demonstration was given in that work for the case of dynamical breaking via condensates, plausibly the result would also apply for models with dynamical axions and very high axion scales.
} 
more, massless (or almost massless) sterile fermions are a low-energy trademark remnant of the massless multiplet that solves the SM strong CP problem.

In order to avoid the $S U\left(3^{\prime}\right)$ sector sourcing back an extra contribution to the strong $\mathrm{CP}$ problem, a minimal extension of its matter sector suffices. Two examples of ultraviolet complete models have been explored in this work: in Model I an extra $S U\left(3^{\prime}\right)$ massless fermion is added, while Model II includes instead a second scalar with the same quantum numbers as the color-unification breaking scalar. From the point of view of the strong CP problem, those two models are very different. Model I features a second dynamical axion with a second PQ scale which is also of order $\Lambda_{\text {diag }}$ and thus low. In Model II, this second PQ scale coincides instead with the much larger color-unification scale, and the associated axion is elementary. We computed the two-loop running of all coupling constants involved, showing that the desired separation of all relevant scales is achieved naturally: a color-unification scale much larger than the two confining ones, $\Lambda_{\text {diag }}$ and $\Lambda_{\mathrm{QCD}}$, and the subsequent separation of the last two. This separation of scales is robust and stable over a wide range of parameter values.

We have found that regardless of the details of the ultraviolet implementation, generically there are the three sources of anomalous currents: the instantons of the confining $S U(3)_{c}$,

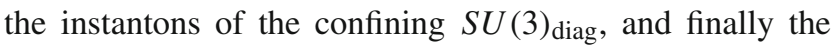
small-size instantons of the spontaneously broken colorunified theory. There are thus three diverse sources of mass for the three pseudoscalars in the theory which couple to anomalous currents: the QCD $\eta^{\prime}$, the dynamical axion inherent to color unification, and the second axion (either dynamical or elementary) associated to the solution of the $\theta^{\prime}$ problem. These three bosons then acquire masses of order $\Lambda_{\mathrm{QCD}}$, $\Lambda_{\text {diag }}$ and $\Lambda_{\text {SSI }}$, respectively, and no standard invisible axion (coupling anomalously only to QCD instantons) is left in the low-energy spectrum. This is generically a very interesting mechanism from the point of view of solving the strong $\mathrm{CP}$ problem with heavy axions and scales around a TeV. The mechanism allows a wide extension beyond the invisible axion range of axion parameter space which solves the strong $\mathrm{CP}$-problem. Typical axion masses are in the $\mathrm{TeV}$ regime and above, although strictly speaking one of the axions can become as light as the usual invisible axion.

With axion scales around the $\mathrm{TeV}$, observable signals at colliders are expected, as well as other rich phenomenology. Generically, the lightest exotic bound states are colored pseudoscalars (QCD octets and in some cases also triplets in Model I, and only octets in Model II). We have recast the results from present experimental searches for heavy colored mesons to infer a $2.9 \mathrm{TeV}$ bound on the confinement scale of the second confining group, $S U(3)_{\text {diag, }}$ which is directly related in Model I to the axion scale.
Overall, Model I may be preferred as: (i) it is exclusively based on solving the strong $\mathrm{CP}$ problem dynamically via massless quarks; (ii) from the point of view of naturalness it does not require any fine-tuning to ensure the hierarchy between the PQ and the electroweak scales, as no PQ field is involved in the scalar potential. In Model II instead, one PQ field participates in the color-unification scalar potential, and furthermore this model is a hybrid dynamical-elementary axion solution to the strong CP problem.

Model $\mathrm{I}$ is also unquestionably safe from the point of view of stability of the axion solution with respect to nonperturbative effects of quantum gravity, as its PQ scales are $\Lambda_{\text {diag }} \sim$ TeV. Furthermore, recent advances suggest that the quantum gravity threat should not be considered a risk even for Model II. The other issue of the cosmological impact of (quasi) stable heavy exotic hadrons and of the (almost) massless sterile fermion remnants can be simply avoided by introducing an inflation scale and reheating temperature lower than $\Lambda_{\text {diag }}$. This last subject deserves future detailed attention in particular in view of the dark matter puzzle.

Acknowledgements We acknowledge M. García Pérez, T. Yanagida, V. Sanz, K. Harigaya, S. Knapen and A. Ringwald for very interesting conversations and comments. M.B.G, R. H., R.dR and P. Q. acknowledge Berkeley LBNL, where part of this work has been developed. This project has received funding from the European Union's Horizon 2020 research and innovation programme under the Marie Sklodowska-Curie grant agreements No 690575 (RISE InvisiblesPlus) and No 674896 (ITN ELUSIVES). M.B.G, R. H., R.dR and P. Q. also acknowledge support from the the Spanish Research Agency (Agencia Estatal de Investigación) through the grant IFT Centro de Excelencia Severo Ochoa SEV-2016-0597. M.B.G, R.dR and P. Q. acknowledge as well support from the "Spanish Agencia Estatal de Investigación" (AEI) and the EU "Fondo Europeo de Desarrollo Regional" (FEDER) through the project FPA2016-78645-P. The work of M.K.G. was supported in part by the Director, Office of Science, Office of High Energy and Nuclear Physics, Division of High Energy Physics, of the U.S. Department of Energy under Contract DE-AC02-05CH11231, and in part by the National Science Foundation under grant PHY-1316783. The work of R. H. was supported to and ESR contract of the H2020 ITN Elusives. The work of P.Q. was supported through a "La Caixa-Severo Ochoa" predoctoral grant of Fundación La Caixa.

Open Access This article is distributed under the terms of the Creative Commons Attribution 4.0 International License (http://creativecomm ons.org/licenses/by/4.0/), which permits unrestricted use, distribution, and reproduction in any medium, provided you give appropriate credit to the original author(s) and the source, provide a link to the Creative Commons license, and indicate if changes were made. Funded by SCOAP ${ }^{3}$.

\section{References}

1. H. Georgi, I.N. McArthur, Instantons and the $u$ quark mass (Harvard University, Preprint). https://lib-extopc.kek.jp/preprints/ PDF/1981/8107/8107158.pdf

2. M. Dine, P. Draper, G. Festuccia, Instanton effects in three flavor QCD. Phys. Rev. D 92(5), 054004 (2015). arXiv:1410.8505 
3. K. Choi, C.W. Kim, W.K. Sze, Mass renormalization by instantons and the strong CP problem. Phys. Rev. Lett. 61, 794 (1988)

4. D.B. Kaplan, A.V. Manohar, Current mass ratios of the light quarks. Phys. Rev. Lett. 56, 2004 (1986)

5. J. Frison, R. Kitano, N. Yamada, $N_{f}=1+2$ mass dependence of the topological susceptibility. PoS LATTICE2016, 323 (2016). arXiv: 1611.07150

6. S. Aoki, Review of lattice results concerning low-energy particle physics. Eur. Phys. J. C 74, 2890 (2014). arXiv:1310.8555

7. S. Aoki, Review of lattice results concerning low-energy particle physics. Eur. Phys. J. C77(2), 112 (2017). arXiv:1607.00299

8. S. Weinberg, Implications of dynamical symmetry breaking. Phys. Rev. D 13, 974-996 (1976). [Addendum: Phys. Rev.D19,1277(1979)]

9. L. Susskind, Dynamics of spontaneous symmetry breaking in the Weinberg-Salam theory. Phys. Rev. D 20, 2619-2625 (1979)

10. S. Dimopoulos, J. Preskill, Massless composites with massive constituents. Nucl. Phys. B 199, 206-222 (1982)

11. K. Choi, J.E. Kim, Dynamical axion. Phys. Rev. D 32, 1828 (1985)

12. R.D. Peccei, H.R. Quinn, CP conservation in the presence of instantons. Phys. Rev. Lett. 38, 1440-1443 (1977). [328(1977)]

13. S. Weinberg, A new light boson? Phys. Rev. Lett. 40, 223-226 (1978)

14. H. Georgi, D.B. Kaplan, L. Randall, Manifesting the invisible axion at low-energies. Phys. Lett. 169B, 73-78 (1986)

15. A. Hook, Anomalous solutions to the strong CP problem. Phys. Rev. Lett. 114(14), 141801 (2015). arXiv:1411.3325 [hep-ph]. http://doi.org/10.1103/PhysRevLett.114.141801

16. H. Fukuda, K. Harigaya, M. Ibe, T.T. Yanagida, Model of visible QCD axion. Phys. Rev. D 92(1), 015021 (2015). arXiv:1504.06084 [hep-ph]. https://doi.org/10.1103/PhysRevD.92.015021

17. Z. Berezhiani, L. Gianfagna, M. Giannotti, Strong CP problem and mirror world: The Weinberg-Wilczek axion revisited. Phys. Lett. B 500, 286-296 (2001). arXiv:hep-ph/0009290 [hep-ph]. https:// doi.org/10.1016/S0370-2693(00)01392-7

18. S.D.H. Hsu, F. Sannino, New solutions to the strong CP problem. Phys. Lett. B 605, 369-375 (2005). arXiv:hep-ph/0408319 [hepph]. https://doi.org/10.1016/j.physletb.2004.11.040

19. C.-W. Chiang, H. Fukuda, M. Ibe, T.T. Yanagida, $750 \mathrm{GeV}$ diphoton resonance in a visible heavy QCD axion model. Phys. Rev. D 93(9), 095016 (2016). arXiv:1602.07909 [hep-ph]. https://doi.org/ 10.1103/PhysRevD.93.095016

20. S. Dimopoulos, A. Hook, J. Huang, G. Marques-Tavares, Acollider observable QCD axion. JHEP 11, 052 (2016). arXiv:1606.03097

21. A. Kobakhidze, Heavy axion in asymptotically safe QCD. arXiv:1607.06552 [hep-ph]

22. P. Agrawal, K. Howe, A Flavorful Factoring of the Strong CP Problem, arXiv: 1712.05803

23. P. Agrawal, K. Howe, Factoring the Strong CP Problem, Submitted to: JHEP (2017) [arXiv: 1710.04213]

24. V.A. Rubakov, Grand unification and heavy axion. JETP Lett. 65, 621-624 (1997). arXiv:hep-ph/9703409

25. A.R. Zhitnitsky, On possible suppression of the axion Hadron interactions (In Russian). Sov. J. Nucl. Phys. 31, 260 (1980). [Yad. Fiz.31,497(1980)]

26. M. Dine, W. Fischler, M. Srednicki, A simple solution to the strong CP problem with a Harmless axion. Phys. Lett. 104B, 199-202 (1981)

27. T. Gherghetta, N. Nagata, M. Shifman, A visible QCD axion from an enlarged color group. Phys. Rev. D 93(11), 115010 (2016). arXiv:1604.01127

28. E. Witten, Current algebra theorems for the U(1) goldstone boson. Nucl. Phys. B 156, 269-283 (1979)

29. G. Veneziano, U(1) without instantons. Nucl. Phys. B 159, 213-224 (1979)
30. P. Di Vecchia, G. Veneziano, Chiral dynamics in the large $\mathrm{n}$ limit. Nucl. Phys. B 171, 253-272 (1980)

31. B. Holdom, M.E. Peskin, Raising the axion mass. Nucl. Phys. B 208, 397-412 (1982)

32. M. Dine, N. Seiberg, String theory and the strong CP problem. Nucl. Phys. B 273, 109-124 (1986)

33. J.M. Flynn, L. Randall, A computation of the small instanton contribution to the axion potential. Nucl. Phys. B 293, 731-739 (1987)

34. C.G. Callan Jr., R.F. Dashen, D.J. Gross, Toward a theory of the strong interactions. Phys. Rev. D 17, 2717 (1978). [36(1977)]

35. G. 't Hooft, Computation of the quantum effects due to a fourdimensional pseudoparticle. Phys. Rev. D 14, 3432-3450 (1976). [70(1976)]

36. M.A. Shifman, A.I. Vainshtein, V.I. Zakharov, Instanton density in a theory with massless quarks. Nucl. Phys. B 163, 46-56 (1980)

37. M.A. Shifman, ed., Instantons in Gauge Theories. 1994

38. C.W. Bernard, Gauge zero modes, instanton determinants, and QCD calculations. Phys. Rev. D 19, 3013 (1979). [109(1979)]

39. F. Sannino, V. Skrinjar, Safe and Free Instantons, arXiv: 1802.10372

40. A. Ringwald, Electroweak instantons/sphalerons at VLHC? Phys. Lett. B 555, 227-237 (2003). arXiv:hep-ph/0212099

41. M. Bauer, M. Heiles, M. Neubert, A. Thamm, arXiv:1808.10323 [hep-ph]

42. The ATLAS collaboration [ATLAS Collaboration], ATLASCONF-2016-070

43. A. Belyaev, G. Cacciapaglia, H. Cai, G. Ferretti, T. Flacke, A. Parolini, H. Serodio, JHEP 1701, 094 (2017). arXiv:1610.06591 [hep-ph]

44. J.R. Ellis, M.K. Gaillard, Strong and weak CP violation. Nucl. Phys. B 150, 141-162 (1979)

45. ATLAS Collaboration, M. Aaboud et al., A search for pairproduced resonances in four-jet final states at $\sqrt{s}=13 \mathrm{TeV}$ with the ATLAS detector. Eur. Phys. J. C 78(3), 250 (2018). arXiv: 1710.07171

46. F. Wilczek, Problem of strong $\mathrm{p}$ and $\mathrm{t}$ invariance in the presence of instantons. Phys. Rev. Lett. 40, 279-282 (1978)

47. D. Goncalves-Netto, D. Lopez-Val, K. Mawatari, T. Plehn, I. Wigmore, Sgluon pair production to next-to-leading order. Phys. Rev. D 85, 114024 (2012). arXiv:1203.6358

48. C. Degrande, B. Fuks, V. Hirschi, J. Proudom, H.-S. Shao, Automated next-to-leading order predictions for new physics at the Lhc: the case of colored scalar pair production. Phys. Rev. D 91(9), 094005 (2015). arXiv: 1412.5589

49. ATLAS Collaboration, M. Aaboud et al., Search for heavy longlived charged $R$-hadrons with the ATLAS detector in $3.2 \mathrm{fb}^{-1}$ of proton-proton collision data at $\sqrt{s}=13 \mathrm{TeV}$, Phys. Lett. B 760, 647-665 (2016), [arXiv:1606.05129]

50. E.W. Kolb, M.S. Turner, The early universe. Front. Phys. 69, 1-547 (1990)

51. K. Choi, K. Kang, J.E. Kim, Cosmological constraints on heavy unstable particles. Phys. Rev. D 32, 2822 (1985)

52. B.A. Dobrescu, The strong CP problem versus Planck scale physics. Phys. Rev. D 55, 5826-5833 (1997). arXiv:hep-ph/9609221

53. H.-Y. Cheng, The strong CP problem revisited. Phys. Rept. 158, 1 (1988)

54. S.M. Barr, D. Seckel, Planck scale corrections to axion models. Phys. Rev. D 46, 539-549 (1992)

55. J. Preskill, S.P. Trivedi, F. Wilczek, M.B. Wise, Cosmology and broken discrete symmetry. Nucl. Phys. B 363, 207-220 (1991)

56. G.R. Dvali, G. Senjanovic, Is there a domain wall problem? Phys. Rev. Lett. 74, 5178-5181 (1995). arXiv:hep-ph/9501387

57. J.E. Kim, A composite invisible axion. Phys. Rev. D 31, 1733 (1985) 
58. R. Holman, S.D.H. Hsu, T.W. Kephart, E.W. Kolb, R. Watkins, L.M. Widrow, Solutions to the strong CP problem in a world with gravity. Phys. Lett. B 282, 132-136 (1992). arXiv:hep-ph/9203206

59. M. Kamionkowski, J. March-Russell, Planck scale physics and the peccei-quinn mechanism. Phys. Lett. B 282, 137-141 (1992). arXiv:hep-th/9202003

60. S. Ghigna, M. Lusignoli, M. Roncadelli, Instability of the invisible axion. Phys. Lett. B 283, 278-281 (1992)

61. H.M. Georgi, L.J. Hall, M.B. Wise, Grand unified models with an automatic Peccei-quinn symmetry. Nucl. Phys. B 192, 409-416 (1981)

62. S.B. Giddings, A. Strominger, Loss of incoherence and determination of coupling constants in quantum gravity. Nucl. Phys. B 307, 854-866 (1988)

63. S.R. Coleman, Why there is nothing rather than something: a theory of the cosmological constant. Nucl. Phys. B 310, 643-668 (1988)
64. G. Gilbert, Wormhole induced proton decay. Nucl. Phys. B 328, 159-170 (1989)

65. S.-J. Rey, The axion dynamics in wormhole background. Phys. Rev. D 39, 3185 (1989)

66. L. Randall, Composite axion models and Planck scale physics. Phys. Lett. B 284, 77-80 (1992)

67. M. Redi, R. Sato, Composite accidental axions. JHEP 05, 104 (2016). arXiv:1602.05427

68. D. Butter, M.K. Gaillard, The Axion mass in modular invariant supergravity. Phys. Lett. B 612, 304-310 (2005). arXiv:hep-th/0502100

69. R. Alonso, A. Urbano, Wormholes and masses for Goldstone bosons. arXiv: 1706.07415 